\title{
Case study: the implementation of a data-driven industrial analytics methodology and platform for smart manufacturing
}

\author{
Peter O’Donovan, Ken Bruton, and Dominic T.J. O’Sullivan \\ IERG, University College Cork, Ireland \\ Corresponding author: peter_odonovan@umail.ucc.ie
}

\begin{abstract}
Integrated, real-time and open approaches relating to the development of industrial analytics capabilities are needed to support smart manufacturing. However, adopting industrial analytics can be challenging due to its multidisciplinary and cross-departmental (e.g. Operation and Information Technology) nature. These challenges stem from the significant effort needed to coordinate and manage teams and technologies in a connected enterprise. To address these challenges, this research presents a formal industrial analytics methodology that may be used to inform the development of industrial analytics capabilities. The methodology classifies operational teams that comprise the industrial analytics ecosystem, and presents a technology agnostic reference architecture to facilitate the industrial analytics lifecycle. Finally, the proposed methodology is demonstrated in a case study, where an industrial analytics platform is used to identify an operational issue in a largescale Air Handling Unit (AHU).
\end{abstract}

\section{INTRODUCTION}

Smart manufacturing refers to a data-driven paradigm that uses real-time pervasive sensor networks, simulation, analytics and robotics, to deliver manufacturing intelligence to every area of the factory (Davis, Edgar, Porter, Bernaden, \& Sarli, 2012; Lee, Lapira, Bagheri, \& Kao, 2013; Lee, 2014; Wright, 2014). Facilities that transition to smart manufacturing operations will be able to address many contemporary operating challenges, such as increasing global competition and rising energy costs, while shortening production cycles and enhancing just-in-time product customization capabilities (Manufacturing et al., 2011; Sharma \& Sharma, 2014). Other potential performance benefits include (1) reduction in capital intensity of 30\%, (2) reduction in product cycle times of up to $40 \%$, and (3) overarching efficiencies across energy, emissions, throughput, yield, waste, and productivity. Extrapolating these efficiencies across entire regions may also derive benefits for the greater economy. Such benefits were

Peter O'Donovan et al. This is an open-access article distributed under the terms of the Creative Commons Attribution 3.0 United States License, which permits unrestricted use, distribution, and reproduction in any medium, provided the original author and source are credited. highlighted in research produced by the Fraunhofer Institute, which estimated the transformation of factories to Industry 4.0 could be worth up to 267 billion Euros to the German economy by 2025 (Heng, 2014). Given the potential benefits that may be derived from smart manufacturing, several government, academic and industry initiatives have emerged in recent years to support its development. Prominent initiatives currently include the Smart Leadership Coalition (SMLC) (Manufacturing et al., 2011), Technology Initiative SmartFactory (Zuehlke, 2010), Industry 4.0 (Lee, Kao, \& Yang, 2014), and The Industrial Internet Consortium (IIC), to name a few.

Modern manufacturing facilities currently employ sensing, control and automation in day-to-day operations (Chand \& Davis, 2010; Davis et al., 2012; Lee et al., 2014). These control and automation technologies deliver operational efficiencies, process innovations, and environmental benefits (Fosso Wamba, Akter, Edwards, Chopin, \& Gnanzou, 2015; Hazen, Boone, Ezell, \& Jones-Farmer, 2014). However, as facilities transition to smart manufacturing, the number of sensors deployed in the factory, as well as the resolution at which they are logging measurements, will inevitably increase (Davis et al., 2012; Lee, Bagheri, \& Kao, 2015; Wright, 2014). These improved sensing capabilities present opportunities to derive new and useful insights from operational data, while also presenting challenges in terms of large-scale data management, processing and analysis (Fosso Wamba et al., 2015; Kumar, Dhruv, Rawat, \& Rathore, 2014; Lee et al., 2013; McKinsey, 2011; Philip Chen \& Zhang, 2014; Verabaquero, Colomo-palacios, \& Molloy, 2014).

Industrial analytics is an important aspect of smart manufacturing, which focuses on the application of datadriven methods and technologies to inform decisionmaking. Some of these data-driven approaches originate from mainstream information technology, before being adapted for industrial use cases. Big Data and Internet of Things (IoT) are good examples of mainstream technologies that have become synonymous with smart manufacturing and industrial analytics. While these (and similar) technologies are central to the development of industrial analytics capabilities, there is an equal, if not greater, dependence on the systematic convergence of teams and 
personnel governing Operation Technology, Information Technology, Data Analytics and Embedded Analytics. However, these multi-disciplinary convergences can be difficult given potential differences in each teams background knowledge, experience and perspective of operating technologies, standards and analytics.

This research presents and applies a formal and systematic methodology to support the development of industrial analytics capabilities. Some aspects of this methodology evolved from previously published research focusing on applications of big data in manufacturing (P. O'Donovan, Leahy, Bruton, \& O'Sullivan, 2015), and factory-to-cloud data integration (P. O'Donovan, Leahy, Bruton, \& O'Sullivan, 2015). The following points highlight contributions from this research, and describe relevant connections with previous research;

- Factory-to-cloud architectures presented in previous research were conceptual. This research extends some of these concepts, and introduces others, to produce a methodology for developing end-to-end industrial analytics capabilities, while also demonstrating and validating the approach using a real-world case study.

- The people and processes needed to implement factoryto-cloud integration, and apply these technologies to problem solving in the factory, were not addressed by previous research. This research places a strong emphasis on identifying operational teams (e.g. Operational and Information Technology), usage scenarios, technical components and systematic processes for end-to-end industrial analytics.

- Low-level details regarding data processing were not addressed in previous research. This research presents a formal taxonomy for cleaning and transforming timeseries industrial data, and a multi-stage data processing workflow pattern for implementation.

- Building and operationalizing analytics models was not addressed in previous research. However, these are fundamental aspects of the industrial analytics lifecycle presented in this research, which identifies the teams, processes, and technologies needed to realize industrial analytics in the factory.

- Finally, ancillary observations from the case study presented in this research (e.g. differing data transfer rates) may prove useful to other researchers implementing industrial analytics. Such observations are sometimes omitted due to publication bias, where seemingly insignificant observations are not reported. Previous research could not report such practical observations due to their conceptual focus.

The remainder of this article is structured as follows Section 2 reviews technologies governing data management across industrial, enterprise and emerging paradigms, Section 3 presents a formal methodology for developing industrial analytics capabilities, Section 4 describes the application of the methodology to the development of an industrial analytics platform in a large-scale manufacturing facility, and Section 5 delivers conclusions from this research.

\section{RELATED WORK}

Many disciplines and technologies are involved in the development of industrial analytics capabilities for smart manufacturing. Developing these capabilities may be viewed as the convergence of traditional Operation and Information Technology, with contemporary data-driven disciplines, such as Big Data Analytics, Machine Learning, Internet of Things (IoT), and Cyber Physical Systems (CPS). The following section reviews prominent technology protocols and architectures related to data transmission, management and processing across Operation Technology, Information Technology and Emerging Technology.

\subsection{Operation Technology}

Operation Technology encompasses control and automation technologies in modern manufacturing facilities (Kastner, Neugschwandtner, Soucek, \& Newman, 2005; Nagorny, Colombo, \& Schmidtmann, 2012; Samad \& Frank, 2007). These technologies typically consist of (1) industrial information systems to inform end-users of operating conditions, (2) Programmable Logic Controllers (PLC) to enact process logic, and (3) field sensors to continuously monitor conditions. The flow of industrial data between these components follows a hierarchical and sequential pattern. Firstly, sensors transmit raw measurements (e.g. room temperature) to PLC's. Secondly, these measurements are persisted in-memory at set intervals (e.g. every 15 minutes). Finally, these in-memory measurements are acquired periodically by industrial information systems to archive measurements in file-based repositories. This hierarchical automation topology may also be designed to comply with control and enterprise standards (e.g. ISA-88, ISA-95) to promote consistency and interoperability (ISA, 2016a, 2016b; Scholten, 2007).

The flow of industrial data in Operation Technology follows two distinct paths - (1) real-time data access via PLC's, and (2) historical data access via file-based archives. Real-time data access may be achieved using standard industrial protocols to interface with PLC's. The most common industrial protocols include Modbus, LonWorks, BACnet, OLE Process Control (OPC), and MT Connect (Alves Santos, Normey-Rico, Merino Gómez, Acebes Arconada, \& de Prada Moraga, 2005; Hong \& Jianhua, 2006; Vincent Wang \& Xu, 2013; Xu, 2012). Historical data access may be achieved using mainstream database and I/O interfaces to interrogate disk-based archives (e.g. CSV, SQL), but the underlying data model (e.g. table structure) of each archive may be entirely proprietary. Such heterogeneous data 
models and technologies can increase the complexity of industrial integration scenarios, given the effort needed to map and integrate their underlying properties.

\subsection{Information Technology}

Information Technology supports business processes using enterprise technologies. Specific applications may include order processing, supply chain management and inventory management, to name a few. These systems may include different architectures and delivery models, ranging from on-premises software, to managed cloud-based solutions. However, contemporary systems tend to embrace Internetoriented architectures and protocols to support pervasive cross-platform accessibility.

Service Oriented Architecture (SOA) is a common design pattern used in modern enterprise systems to support interactions between distributed components (Al-jaroodi \& Mohamed, 2012; Cardiel, Gil, Somolinos, \& Somolinos, 2012; Gligor \& Turc, 2012). SOA provides a technology agnostic means of exposing system functionality as autonomous services, which may be consumed by distributed components. These autonomous services are commonly implemented as Web Services, which use Internet standards and protocols to invoke actions on remote machines. The use of such standards can facilitate interoperability across a wide-range of platforms and devices, which is of particular importance to modern enterprise systems, where prescribing the use of platforms and devices may not be possible due to geographically distributed users and technology preferences. There are currently two main approaches used to develop Web Services, namely Representational State Transfer (REST) and Simple Object Access Protocol (SOAP). While REST is an architectural style that employs Hypertext Transfer Protocol (HTTP) to initiate services across distributed networks, SOAP is an XML-based message protocol for exchanging information and invoking services on distributed computers. Both approaches are technology neutral, meaning they can be developed using different programming languages and technologies (e.g. Java, .NET).

\subsection{Emerging Technology}

In recent years, contemporary technology paradigms closely related to smart manufacturing have emerged (e.g. cloud manufacturing and cyber manufacturing). These paradigms describe connected industrial enterprises, where Operation Technology and Information Technology have converged. This convergence is necessary to support the formation of data-rich production environments with pervasive sensing and analytics capabilities. These paradigms commonly embrace mainstream technologies (e.g. Cloud Computing, Big Data and Internet of Things) to address traditional industrial engineering challenges and scenarios (e.g. process improvement, equipment maintenance, and energy optimization) (O'Donovan et al., 2015).

Manufacturing technology paradigms that employ cloud computing typically use service-orientation (e.g. SOA) to share industrial resources and utilities, thereby centralizing and consolidating efforts across geographically distributed processes and operations (Vincent Wang \& Xu, 2013; Wu, Greer, Rosen, \& Schaefer, 2013; Xu, 2012). These services typically reside in the cyber world (i.e. cloud), which embodies the data storage and compute power needed to process complex models and simulations, while these analytical results are relayed to the physical world (i.e. factory) to inform decision-making. Those systems that facilitate interactions between cyber and physical worlds are commonly referred to as Cyber Physical Systems (CPS), which is a multidisciplinary field of research that intersects Engineering, Big Data, Internet of Things, and Machine Learning (Bagheri, Yang, Kao, \& Lee, 2015; Dworschak \& Zaiser, 2014; Lee, Ardakani, Yang, \& Bagheri, 2015; Lee, Bagheri, et al., 2015; Wright, 2014). The main impediment facing the adoption of smart manufacturing technologies relates to legacy issues, such as poor interoperability, proprietary standards, and inadequate data management. Those facilities migrating to smart manufacturing must focus on employing open and consistent standards (Brandl, 2008; Chungoora et al., 2013; Emerson, Kawamura, \& Matthews, 2007; Giovannini, Aubry, Panetto, Dassisti, \& El Haouzi, 2012; Reinisch, Granzer, Praus, \& Kastner, 2008; Scholten, 2007; Weiss \& Donmez, 2014).

Although some existing applications and architectures may support elements of industrial analytics, broader methodologies are needed to illustrate the roles, components and processes in the industrial analytics lifecycle. To address these needs, this research presents and applies an industrial analytics methodology that describes a closedloop industrial analytics lifecycle, beginning with the classification of operational teams, and culminating in the operationalization of embedded analytics in real-time factory operations.

\section{INDUSTRIAL ANALYTICS METHODOLOGY}

This section presents a methodology to support the development of industrial analytics capabilities in largescale manufacturing facilities. The phases of the methodology are illustrated in Figure 1. Firstly, the technology and analytics teams are formed, and assigned specific roles and responsibilities. Secondly, an information system architecture is implemented to enable operational data in the factory to automatically flow between each team. Finally, operational questions are identified and answered using a formal analytics process. The following sections describe the methodology's theoretical concepts using technology agnostic, formal and systematic methods. 


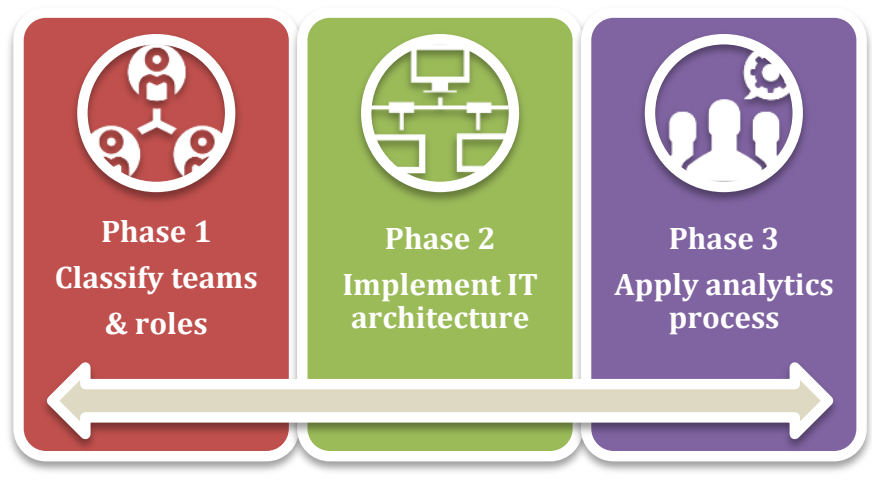

Figure 1. Phases of industrial analytics methodology

\subsection{Phase 1 of 3 - Industrial Analytics Teams}

Smart manufacturing requires the convergence of Operation and Information Technology to produce seamless streams of operational intelligence in the factory. In terms of industrial analytics, the convergence of contemporary analytics teams must also be considered. Therefore, four teams are included in the proposed industrial analytics lifecycle - (1) Operation Technology, (2) Information Technology, (3) Data Analytics, and (4) Embedded Analytics. Figure 2 illustrates the main relationships that exist between teams, responsibilities and standards. Firstly, all teams must commit to the adoption of technology standards in their respective environments (e.g. automation and control, cloud, analytics etc.). Secondly, teams are broadly classified as those responsible for data integration, processing and management, and those responsible for building and operationalizing analytics. Thirdly, collaborative inter-team relationships follow a clockwise direction, from Operation Technology through to Embedded Analytics.

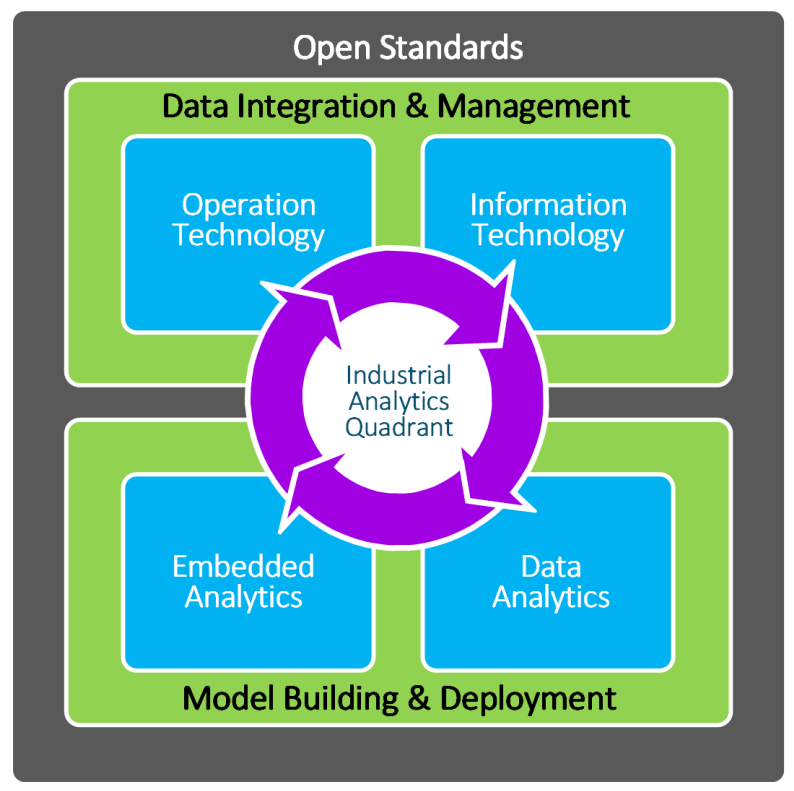

Figure 2. Industrial analytics quadrant
The roles and responsibilities of each team in the industrial analytics lifecycle methodology are summarized in the following sections.

\subsubsection{Operational Technology Team}

Operational Technology teams ensure industrial information and automation systems are operating as intended. In the industrial analytics lifecycle, they guide the integration and management of industrial data from systems and devices in the factory. Their most important relationship is with Information Technology, with whom they must collaborate to construct end-points that connect the factory to the global information system architecture that supports the industrial analytics lifecycle.

\subsubsection{Information Technology Team}

Information Technology teams fulfill a wide-range of roles across the enterprise, including the provisioning of compute resources, software development, business intelligence, data management and integration. In the industrial analytics lifecycle, they are needed to develop the information system architecture and tools that facilitate the seamless flow and processing of operational data. They must communicate with each team to ensure the appropriate compute resources, software and tools are available.

\subsubsection{Data Analytics Team}

Data Analytics teams employ data-driven methods and tools (e.g. machine learning) to derive insights that can positively impact operations. In the industrial analytics lifecycle, they are needed to build accurate data-driven models that support decision-making processes. Their most important relationship is with Embedded Analytics, with whom they must collaborate to (a) elicit and comprehend useful operational questions, and (b) ensure model outputs are contextually accurate.

\subsubsection{Embedded Analytics Team}

Embedded Analytics teams develop applications and tools that are deployed in the factory to inform real-time decisionmaking. In the industrial analytics lifecycle, they use domain expertise to identify and inform high-impact analytics questions, as well as operationalizing productionready analytics models in factory operations. They have important relationships with both Data Analytics and Operation Technology. They utilize knowledge of factory operations to ensure Data Analytics are investigating relevant and useful questions, while they collaborate with Operation Technology to ensure real-time data streams are accessible to embedded applications.

The teams and roles in the industrial analytics lifecycle were discussed in this section to provide clear boundaries for those contributing to the development of industrial analytics 
capabilities. The next section focuses on technical responsibilities (e.g. data integration, processing and modeling) associated with each team.

\subsection{Phase 2 of 3 - Industrial Analytics Reference Architecture}

Figure 3 illustrates a technology-agnostic reference architecture depicting an industrial analytics lifecycle. The architecture incorporates the technology and analytics teams described previously, as well as primary technical components, and industrial data streams, which are needed to support collaboration, interoperability and computation. These details provide the theoretical basis for developing an industrial analytics lifecycle, without being overly prescriptive or immutable. The architecture should be considered an open and technology neutral artifact, which can be extended or modified to meet the needs of particular facilities. Table 1 summarizes the dimensions of the reference architecture.

\begin{tabular}{l|l}
\hline Dimension & Description \\
\hline Data Streams & $\begin{array}{l}\text { Batch and real-time data streams are shown } \\
\text { at the top of the reference architecture, with } \\
\text { each part of the industrial analytics lifecycle } \\
\text { grouped under one of these streams. The } \\
\text { left side of the reference architecture } \\
\text { illustrates how batch data is propagated to } \\
\text { Data Analytics to build data-driven models, } \\
\text { while the right side illustrates real-time data } \\
\text { being consumed in the factory. }\end{array}$ \\
\hline Lifecycle Stage & $\begin{array}{l}\text { Each stage in the industrial analytics } \\
\text { lifecycle is represented by technology and } \\
\text { analytics teams. They function as containers } \\
\text { for technical components to ingest, prepare, } \\
\text { analyze and operationalize data-driven } \\
\text { models. Stages are linked as per the }\end{array}$ \\
industrial analytics quadrant from the \\
previous phase - (1) Operation Technology, \\
(2) Information Technology, (3) Data \\
Analytics, and (4) Embedded Analytics.
\end{tabular}

Table 1. Reference architecture dimensions
The technology and analytics teams are deliberately analogous with each stage in the reference architecture. This provides a consistent vocabulary to convey classifications and groupings throughout the methodology. The technical components and functions in each stage of the reference architecture are discussed in the following sections.

\subsubsection{Operation Technology Components}

Technical components in the Operational Technology stage archive operational data and establish communications with Information Technology. This is illustrated in the reference architecture, where operational data from a PLC in the realtime stream is periodically archived by the Building Management System. These archives (e.g. log files) are stored on disk, which enables ingestion components to access and transmit historic operational data to a centrally accessible data lake. While systems and storage formats may change from factor-to-factory, the process of archiving and accessing operational data should be similar.

Building analytics models that answer operational questions are largely dependent on the availability of high-quality training data, therefore, components at the Operation Technology stage are crucial to the industrial analytics lifecycle. Of the components depicted in the reference architecture, industrial information systems and data archives are almost certain to exist in modern manufacturing facilities. Where these components do not exist, it is the responsibility of Operation Technology to implement solutions that archive operational data from across the factory. In contrast, data ingestion components and communication endpoints may not exist given the traditional separation between Operation and Information Technology. Where these components do not exist, Operation and Information Technology must collaborate to agree specifications and protocols, with Information Technology taking responsibility for implementation and deployment. 




Figure 3. Industrial analytics lifecycle and reference architecture

\subsubsection{Information Technology Components}

Technical components in the Information Technology stage are primarily used to store, process and prepare operational data transmitted from Operation Technology. These components collaborate to automate the delivery of analytics-ready data, which may be consumed by other stages in the industrial analytics lifecycle. The reference architecture illustrates the interactions between these components using the example of data from an Air Handling Unit (AHU). Initially, AHU data transmitted from Operation Technology is tagged and stored in the data lake. This triggers a call to the workflow engine, which searches for workflows that can clean and process AHU data. Where a suitable workflow is identified, the workflow engine constructs and executes a data processing job, and provides the transmitted AHU data as input. These jobs consist of multiple modules, where each module performs a single operation on the AHU data (e.g. sort by timestamp, remove duplicates etc.) to produce an analytics-ready data set.

The Information Technology stage has three communication endpoints that connect to Operation Technology and Data
Analytics in the industrial analytics lifecycle. As previously mentioned, the inbound endpoint for Operation Technology facilitates the transmission of data from the factory, while a second inbound endpoint for Data Analytics enables those undertaking analysis to access the final analytics-ready data set from workflows (e.g. AHU workflow), or data output from a particular workflow stage. To illustrate the usefulness of this design, consider a scenario where the final output from a particular workflow aggregates a time-series using daily averages, but analytical questions later arise that require clean 15-minute resolution data. By following the multistage approach, the 15 -minute data may be obtained from an earlier stage in the workflow, before daily average calculations were applied. This illustrates how reusable and accessible workflows can reduce duplicated effort for data cleaning and transformation operations.

Information Technology components illustrated in the reference architecture may exist in facilities where cloudbased big data infrastructures have been adopted. However, where these components exist in a business enterprise context, they may require some amendments to work with industrial operational data (e.g. time-series). In facilities 
where these components do not exist, Information Technology should be responsible for their design, development and implementation. These components are needed to abstract Data Analytics from time-consuming and complex processing of ad hoc and proprietary operational data, while also being critical to the scalability and resilience of the industrial analytics lifecycle.

The workflows depicted in the reference architecture comprise multiple data processing modules, which are positioned in a particular order to produce an analyticsready dataset. Such datasets are commonly referred to as tidy data sets, where each column refers to a single variable/feature/measurement, and each row refers to a single observation at a point in time. Table 2 describes different types of processing modules that may be included in a workflow. These classifications provide a common vocabulary for common industrial time-series processing patterns. Each module accepts one or more files as input, and produces a single file as output. This ensures consistency in module implementations, while also enabling modules to be 'chained' (i.e. output from one module may be used as input for the next module). Finally, enforcing an input/output interface also enables modules to be easily substituted (e.g. replaced) without breaking workflows.

\begin{tabular}{|c|c|}
\hline Name & Description \\
\hline Type 1 (Normalizer) & $\begin{array}{l}\text { Modules of type Normalizer take } \\
\text { proprietary files from the data lake as } \\
\text { input and wrangle them in to a basic } \\
\text { time-series format consisting of } \\
\text { timestamp and value columns. }\end{array}$ \\
\hline Type 2 (Mapper) & $\begin{array}{l}\text { Modules of type Mapper rename log } \\
\text { files to give them context, as many } \\
\text { raw log files in facilities use arbitrary } \\
\text { or auto-generated names. Although } \\
\text { renaming files only provides a } \\
\text { minimal level of contextualization, it } \\
\text { enables end-users and other processes } \\
\text { to identify data by name. }\end{array}$ \\
\hline Type 3 (Aggregator) & $\begin{array}{l}\text { Modules of type Aggregator merges } \\
\text { individual time-series log files to a } \\
\text { single analytics-ready file. }\end{array}$ \\
\hline Type 4 (Mutator) & $\begin{array}{l}\text { Modules of type Mutator are used to } \\
\text { transform any aspect of an analytics- } \\
\text { ready file. This includes deriving and } \\
\text { appending new data (e.g. columns), or } \\
\text { undertaking fundamental cleaning and } \\
\text { transformation operations on existing } \\
\text { data (e.g. percentage to decimal). }\end{array}$ \\
\hline Type 5 (Action) & $\begin{array}{l}\text { Modules of type Action undertake ad } \\
\text { hoc routines/tasks when a workflow } \\
\text { has been completed. This may include } \\
\text { tasks such as transmitting the output } \\
\text { to another database or system. }\end{array}$ \\
\hline
\end{tabular}

Table 2. Classes of workflow processing modules
When constructing a workflow consisting of multiple processing modules, some guidelines relating to the position and order of modules must be adhered to. These guidelines are described below;

- The first processing module in a workflow should be of type Normalizer. Such modules are generally specific to the information system or repository from which the data originated. In the reference architecture example, the first Normalizer module in the AHU workflow may be responsible for transforming the BMS data to a generic time-series representation.

- The second processing module in a workflow should be of type Mapper. This facilitates the labeling and high-level contextualization of the data being processed. In the reference architecture example, the second Mapper module in the AHU workflow may rename arbitrary log files using the sensor measurements being monitored in the AHU.

- The third processing module in a workflow should be of type Aggregator. These modules produce a tidy data set by aggregating data from multiple log files. In the reference architecture example, the third Aggregator module in the AHU workflow may aggregate all measurements for a particular AHU to present them in a single file.

- Processing modules of type Mutator can be positioned anywhere after the third module. Each Mutator module implements a single processing function. Therefore, where several data transformations must be applied to data in a workflow, the equivalent number of Mutator modules should be present. Similar to good software design principles, singularity and modularity can be used to promote reuse, while reducing maintenance.

- Finally, modules of type Action can be used to execute triggers, such as emailing a notification or building a PDF report. Given these modules do not output a file that can be used by other modules, Action modules may only be positioned as the last module in a workflow.

Given its intermediary role between Operation Technology and Data Analytics, coupled with the responsibility of providing data management and processing for the factory's operational data, Information Technology may represent the most complex and time-consuming aspect of the industrial analytics lifecycle. To reduce this complexity, the reference architecture illustrates the operation of a prescriptive cloudbased workflow pattern, which includes a formal taxonomy that classifies time-series processing routines. 


\subsubsection{Data Analytics Components}

Technical components in the Data Analytics stage use datadriven methods to derive insights that can positively affect operations. This requires Data Analytics personnel and components to acquire analytics-ready operational data from Information Technology, build insightful data-driven models, and support the deployment of these models in the factory. The reference architecture illustrates an example of an R Console accessing and exploring data from an AHU workflow, before building, standardizing and operationalizing an AHU model (e.g. issue identification) to a real-time stream in the factory.

The Data Analytics stage has three communication endpoints that connect to Information Technology and Embedded Analytics. These endpoints consist of two outbound channels, and one inbound channel. The outbound endpoint to Information Technology facilitates the acquisition of analytics-ready data from workflows, while the outbound endpoint to Embedded Analytics supports the deployment of production-ready models to real-time streams in the factory. The inbound endpoint from Information Technology may be used to automatically retrain existing data-driven models when new training data becomes available. These components facilitate turnkey data analysis, model building, standardization and deployment in the industrial analytics lifecycle, without having to focus on low-value, complex and time-consuming activities (e.g. data integration and cleaning).

Some components illustrated in the reference architecture may already exist in facilities where statistical data analysis is used for business intelligence or reporting. Examples of components may include those relating to statistical software applications, such as R and SAS. The components relating to building, training and operationalizing datadriven models are less likely to exist, but may be present in facilities currently adopting aspects of smart manufacturing or advanced analytics. Where these components do not exist, Data Analytics and Information Technology must collaborate to define requirements and specifications, with Information Technology leading implementation. Unlike other stages in the reference architecture, Data Analytics and Embedded Analytics stages prescribe the use of Predictive Modeling Markup Language (PMML) (Data Mining Group, 2016) to encode and standardize data-driven models. This aspect of the reference architecture may be abstracted in future iterations when there are well-known alternatives to PMML.

\subsubsection{Embedded Analytics Components}

Technical components in the Embedded Analytics stage facilitate the operationalization of production-ready datadriven models, which enables outputs from Data Analytics to positively affect real-time operations. Where analytics models are not operationalized, the valuable data insights they provide are inaccessible to decision-making processes in the factory. The reference architecture illustrates an example of an embedded application in the factory, which acquires real-time AHU measurements from a PLC, before transmitting these measurements to a Scoring Engine for evaluation. The Scoring Engine passes these measurements to the PMML encoded AHU model to derive a result (i.e. issue identification), which is relayed back to the embedded application to take appropriate action.

The Embedded Analytics has two communication endpoints that connect to Data Analytics and Operation Technology. These endpoints consist of one inbound channel, and one outbound channel. The inbound endpoint from Data Analytics supports the real-time deployment of data-driven models, while the outbound endpoint to Operation Technology provides embedded applications with access to continuous real-time measurements. While some types of embedded applications are likely to exist in modern manufacturing facilities, where closed or proprietary implementations have been employed, their inclusion in the industrial analytics lifecycle may not be viable. Generally, these embedded applications are associated with a particular aspect of the manufacturing domain (e.g. issue identification, maintenance, scheduling etc.).

The subject matter experts in Embedded Analytics are needed to identify areas where analytics may have the greatest impact on operations. This knowledge should inform the development of analytics questions, and validate the accuracy of models before they are used in embedded analytics applications. Given these applications depend on PMML and Scoring Engine components to produce data intelligence, Embedded Analytics must collaborate with Information Technology to ensure their availability.

The reference architecture presented in this phase of the methodology provides a formal and consistent view of an industrial analytics lifecycle. Developing a more prescriptive architecture is difficult considering the possible technology permutations that may exist from factory-tofactory. Hence, the level of abstraction upon which the reference architecture is modeled aims to balance technology neutrality and high-level specification. This provides flexibility around technology selection, but exposes the risk that misinterpretation of the lifecycle may lead to inappropriate technology choices. While previous phases of the methodology described operational and technical components in the industrial analytics lifecycle, the next phase presents an analytics process that demonstrates how the lifecycle may be used to investigate an industrial engineering problem.

\subsection{Phase 3 of 3 - Industrial Analytics Process}

This phase of the methodology describes a sequential process for applying the industrial analytics lifecycle to operational challenges. Each step in the process prescribes 
an action to be undertaken, as well as identifying teams responsible for their execution. This serves to coordinate workloads across multi-disciplinary teams, while ensuring efforts are guided by well-defined objectives. Furthermore, this formal and systematic approach may reduce the risk of project failure by clearly apportioning responsibilities and actions, while also facilitating the early identification of critical issues (e.g. data availability). Figure 4 illustrates each step in the industrial analytics process.

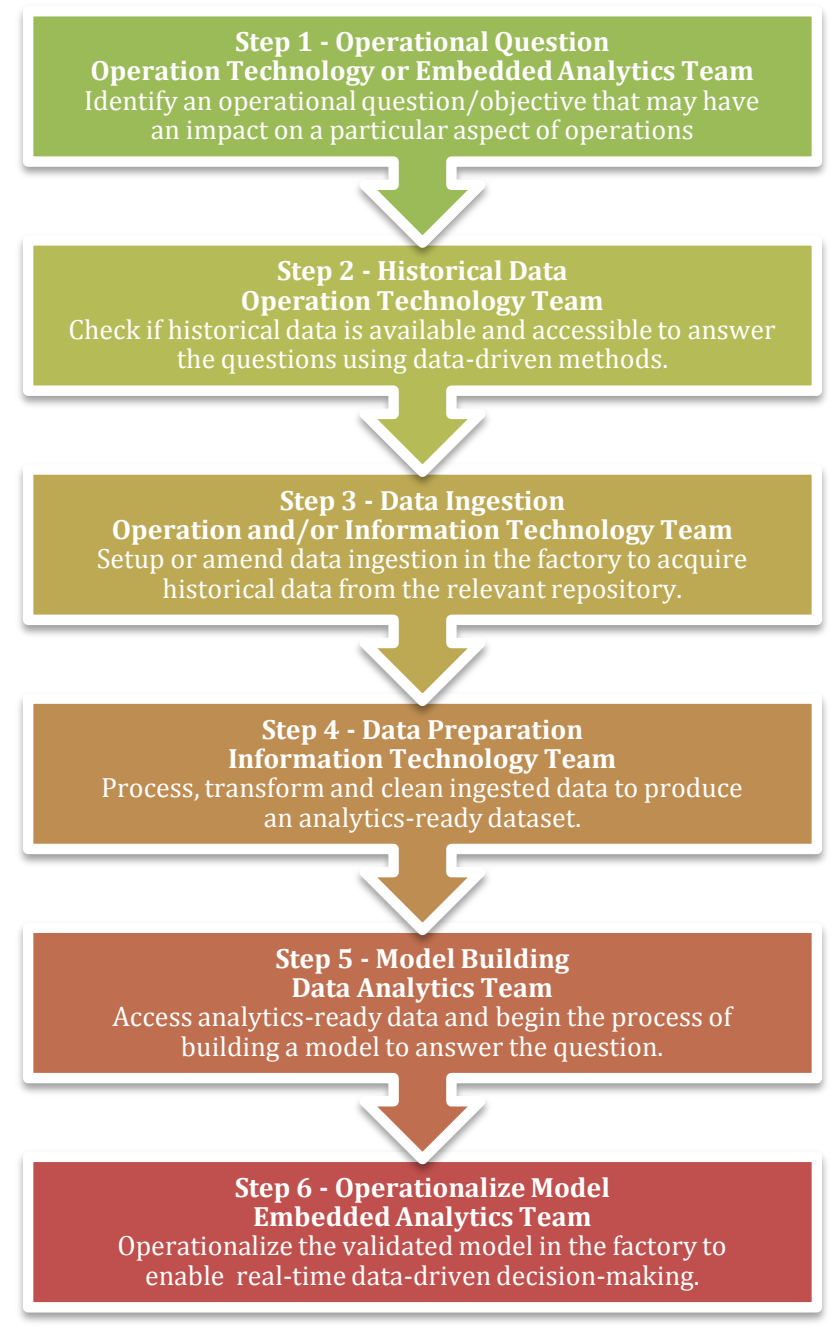

Figure 4. Industrial analytics process steps

The process does not currently provide conditional paths to support alternative actions, which may be necessary in scenarios where a particular step could not be completed. For example, it would not be possible to complete Step 3 (i.e. data ingestion) without access to historical data. In this particular example, an alternative action may involve Operation Technology creating a new data archive. While future iterations of this process may include conditional paths, the current process depends on teams encountering issue(s) using their discretion to either (a) fix the issue(s), or (b) terminate the process. This industrial analytics process is used to structure the following case study, which focuses on the application of the industrial analytics methodology to identify operating issues in a large-scale industrial AHU.

\section{Case Study}

This case study applies the industrial analytics methodology to an issue identification scenario in a large-scale manufacturing facility in Cork, Ireland. The objective of the study was to evaluate the methodology, and implement technology components in the reference architecture to form an industrial analytics lifecycle. This required the use of several technologies and platforms, which were chosen at different points in the process. However, technology decisions described in this study should not be considered a prescribed technical implementation. Indeed, changes in the manufacturing environment, available resources, or engineering application, may have resulted in completely different implementation decisions.

\subsection{Step 1 - Operational Question}

The operational question of this study focuses on issue identification in large-scale industrial Air Handling Units (AHU's). Given the facility used in this study is subject to regulation and quality control, the analysis did not include AHU's subject to quality assessment and validation. The rationale for choosing $\mathrm{AHU}$ issue identification as the operational question is provided below;

- The study's purpose is to demonstrate and validate the industrial analytics methodology. Therefore, operational questions should avoid potential impediments, such as restricted access stemming from quality policies or procedures.

- Although the potential impact of the question is not central to this study, it should be a real operating problem that can be solved within the scope of the study, while providing the opportunity for further investigation.

- Given the proposed methodology demands diverse skills and knowledge to execute an industrial analytics lifecycle, the operational question should align with the skills and knowledge of the authors.

Once issue identification for AHU's was agreed as the guiding operational question, the next step was to identify a repository of AHU data that could be used to build a datadriven model.

\subsection{Step 2 - Historical Data}

Component-level sensors in AHU's may be used to predict system health and energy inefficiencies. Examples of such measurements include mechanical component positions, temperature, and airflow. These measurements are typically 
transmitted in real-time across automation and control networks, while they may also be periodically archived as disk-based repositories. In this study, an on-premises Cylon Building Management System (BMS) was identified as the main source of energy data. Automatic archiving of this data executed at 5AM each day. During the archival process, CSV log files were appended with data from the previous 24-hour period, with each file containing historical measurements for a single sensor. Brief investigations of other on-premises BMS's confirmed that archival processes across vendors are similar, but $\log$ file formats and data models output from these processes varied significantly.

The identified Cylon BMS archive was explored to confirm the availability of AHU data, as well as compiling metadata to characterize the archive. Firstly, we randomly chose three AHU's to investigate. Of those units, we decided to use the AHU with the most historical data as the subject for analysis in this study. This unit was labeled AHU9 and provided access to 4 years of 15-minute measurements. Secondly, the BMS archive was analyzed to produce metadata from the archive's data properties. The archive consumed 1.06 GB of disk space on the BMS PC. This consisted of 838 log files, with each storing historical measurements for a single sensor. The timespan of measurements in each file ranged from a couple of months to 4 years. The largest file in the archive measured $13 \mathrm{MB}$, while the smallest file measured a mere $1 \mathrm{~KB}$. Approximately 5\% of all $\log$ files were larger than $5 \mathrm{MB}$, which loosely represented files with 4 years of data. Log files smaller than $1 \mathrm{MB}$ were indicative of (a) new sensors that recently began archiving, or (b) legacy sensors where archiving was disabled. However, file size could not be used to accurately predict the range of measurements in a $\log$ file. For example, two log files measuring 5MB and $12 \mathrm{MB}$ may contain the same date ranges and measurement resolutions, but the latter may contain higher precision readings, which simply consumes more Bytes on disk.

The identification and verification of historical AHU data to support issue identification analysis was completed in this step. The next step focused on the development of a data ingestion process that integrated this historical data in a centrally accessible cloud-based repository.

\subsection{Step 3 - Data Ingestion}

Amazon Web Services (AWS) was chosen as the cloud platform to host Information Technology components. Of those components, the data lake was initially required to support data ingestion. The data lake was implemented using the Simple Storage Service (S3), which provided scalable and fault tolerant file storage, while providing an Application Programming Interface (API) for factory-tocloud communication. This API was integrated with a purpose-built data ingestion application written in C\# .NET to continuously stream energy data to the data lake.
The ingestion process was tested on different computers and networks to reduce potential technology biases (e.g. high bandwidth). First, the process was executed on a development $\mathrm{PC}$ with diagnostic tools enabled to monitor resource usage. The development PC specification included an Intel Core i5-4380U CPU @ 2.80GHz processor, 4 GB memory, and $200 \mathrm{~GB}$ solid-state hard drive, running on Windows 8.1 Enterprise. Bandwidth availability was measured at $40 \mathrm{Mbps}$ download and $10 \mathrm{Mbps}$ upload. Figure 5 and Figure 6 show CPU and Memory profiles recorded during execution. Both profiles demonstrate utilization was low relative to available compute resources, with the process execution time taking approximately 11 minutes. Second, after testing, validating and profiling the process in the development environment, the application was deployed to the BMS PC in the factory. The BMS PC specification included an Intel Core 2 Duo E8400 @ 3.00GHz, 2GB memory, 500GB hard drive, running on Windows XP Professional with Service Pack 3. Bandwidth availability was measured at 55Mbps download and $4 \mathrm{Mbps}$ upload. The execution time of the process in the facility was approximately 39 minutes. Given diagnostic tools were not available on the BMS PC, execution time was derived programmatically by recording start and finish times.

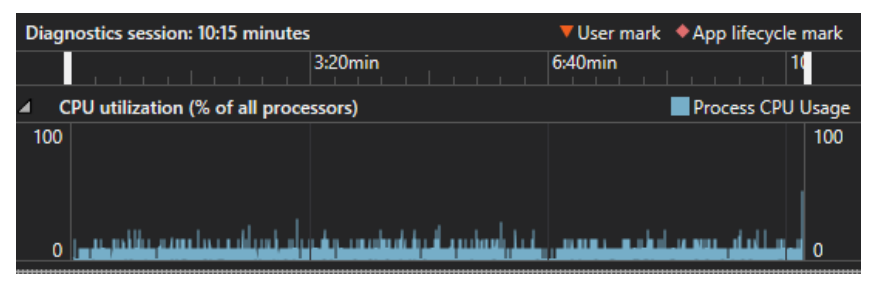

Figure 5. CPU profile for BMS archive ingestion

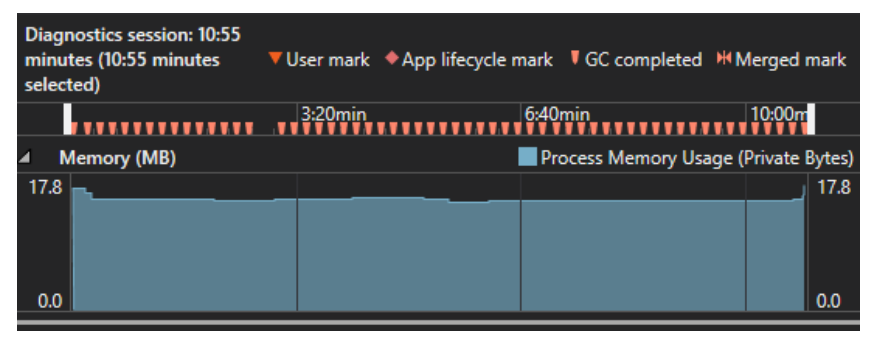

Figure 6. Memory profile for BMS archive ingestion

Given the satisfactory performance of the data ingestion application during testing, the application was setup as a scheduled task to execute at 9AM each day on the BMS PC. This provided the existing BMS archival process with a 4hour window to collect the previous days data, and refresh its data archive. When triggered by the scheduled task, the ingestion application read an XML-based configuration file to acquire endpoints and credentials for the data lake, as well as constructing contextual tags to label data (e.g. energy data from Site $N$ ). Figure 7 presents a screenshot of the configuration parameters used to ingest energy data, while Table 3 provides a summary of these parameters. 


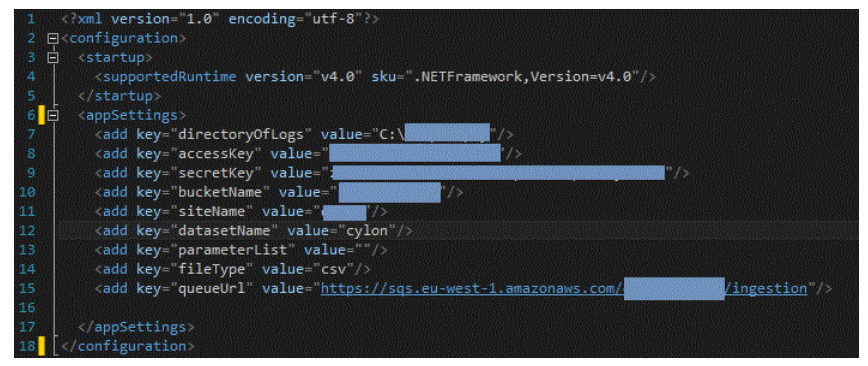

Figure 7. Configuration for BMS archive ingestion

\begin{tabular}{l|l}
\hline Parameter & Description \\
\hline directoryOfLogs & $\begin{array}{l}\text { Local or network directory path that } \\
\text { contains the log files. }\end{array}$ \\
\hline accessKey & $\begin{array}{l}\text { First part of an AWS credential that } \\
\text { identifies the facility from which data is } \\
\text { being ingested. }\end{array}$ \\
\hline secretKey & $\begin{array}{l}\text { Second part of an AWS credential that } \\
\text { identifies the facility from which data is } \\
\text { being ingested. }\end{array}$ \\
\hline bucketName & $\begin{array}{l}\text { Static label to name the industrial } \\
\text { analytics platform. }\end{array}$ \\
\hline siteName & $\begin{array}{l}\text { Human-readable name of the facility } \\
\text { from which data is being ingested - this } \\
\text { forms part of a contextual tag for } \\
\text { identifying datasets. }\end{array}$ \\
\hline datasetName & $\begin{array}{l}\text { Human-readable name of the dataset } \\
\text { being ingested - this forms part of a } \\
\text { contextual tag for identifying datasets. }\end{array}$ \\
\hline parameterList & Limits ingestion to the listed files. \\
\hline fileType & Limits ingestion to a particular file type. \\
\hline queueUrl & $\begin{array}{l}\text { Specifies the Information Technology } \\
\text { endpoint for transmitting data. }\end{array}$
\end{tabular}

Table 3. Data ingestion configuration parameters

\subsection{Step 4 - Data Processing}

Given the availability of energy data in the data lake, Simple Queue Services (SQS) was used to orchestrate data processing across workflows using a publish/subscribe pattern, with ElasticBeanstalk used to host individual processing modules in each workflow. The processing instructions for each workflow were stored in SQL Relational Database Service (RDS) to control execution, while each cloud-based service was configured with autoscaling capabilities to support on-demand acquisition of compute resources for large-scale processing. Figure 8 illustrates the database schema used to configure workflows and components. This schema illustrates Workflows are comprised of multiple stages, with each stage connected to Processors (i.e. processing modules) that represent the type of data manipulation to be performed.

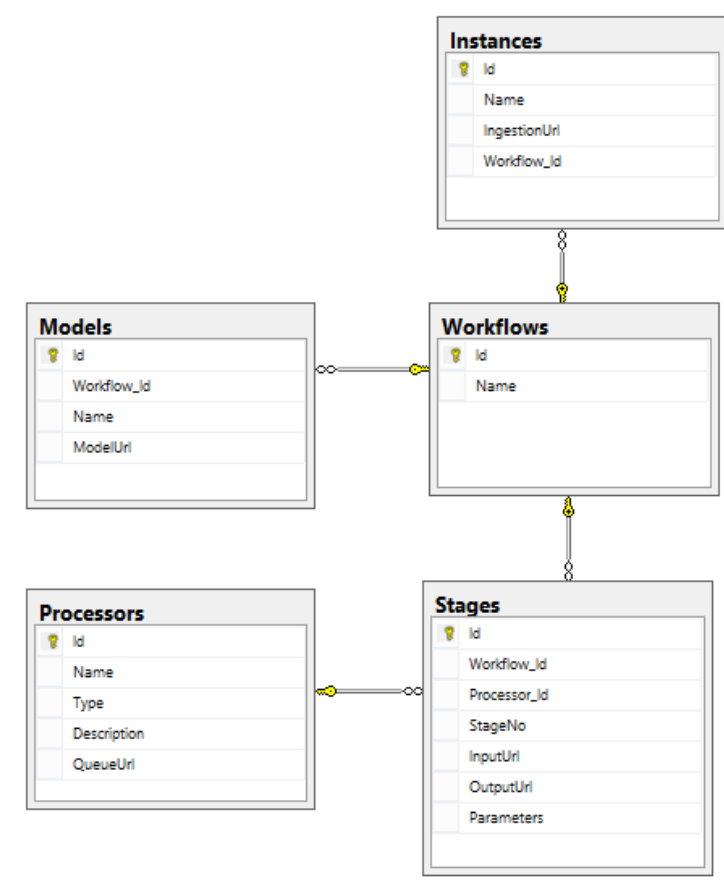

Figure 8. Database schema for workflow management

Figure 9 illustrates the publish/subscribe processing pattern for the AHU workflow. Each stage in the workflow is associated with a (a) message queue to receive instructions, (b) background data processing module, and (c) storage repository to persist output. Figure 10 illustrates processing modules implemented in this study, and their relationship to the processing taxonomy from the methodology. These processing modules are summarized in Table 4 .

\begin{tabular}{l|l|l}
\hline Type & Name & Description \\
\hline Parser & cylon-log & $\begin{array}{l}\text { Transforms Cylon log files to a } \\
\text { basic time-series format with } \\
\text { timestamp and value. }\end{array}$ \\
\hline Mapper & ahu-points & $\begin{array}{l}\text { Renames the auto-generated } \\
\text { filenames using a convention } \\
\text { for AHU instrumentation. }\end{array}$ \\
\hline Aggregator & time-series & $\begin{array}{l}\text { Merges log files for AHU } \\
\text { instrumentation and writes } \\
\text { contents to a single file. }\end{array}$ \\
\hline Mutator & ahu- delta & $\begin{array}{l}\text { Derives the AHU's operational } \\
\text { mode for each instance. }\end{array}$ \\
\hline Mutator & ahu-labels & $\begin{array}{l}\text { acrives temperature differences } \\
\text { and appends the new data to the } \\
\text { existing data set. }\end{array}$ \\
\hline $\begin{array}{l}\text { Appends a classification label } \\
\text { for AHU diagnoses to enable } \\
\text { the data set to be used as } \\
\text { training data for models. }\end{array}$
\end{tabular}

Table 4. Implemented AHU workflow modules 


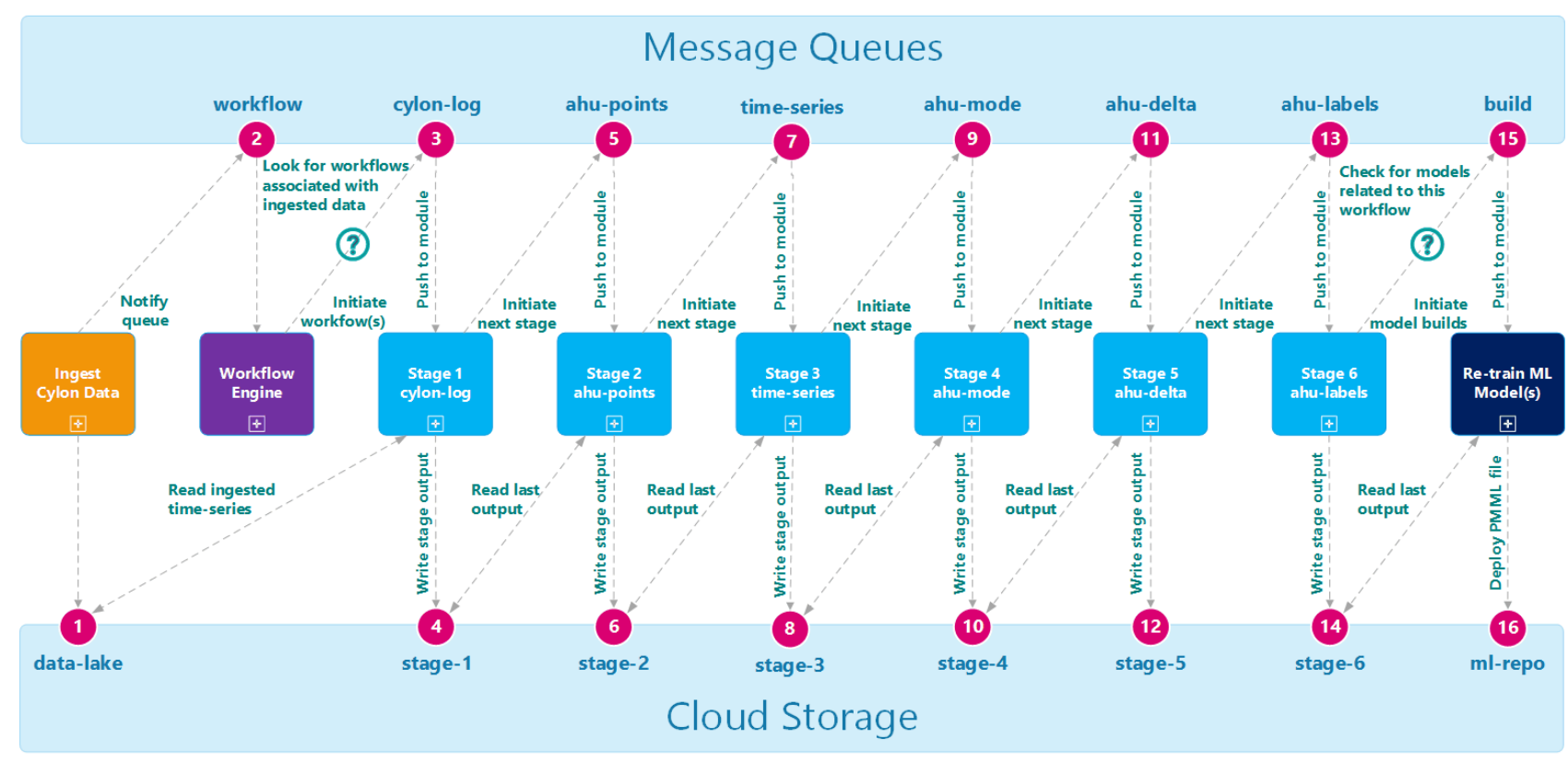

Figure 9. Implemented AHU workflow

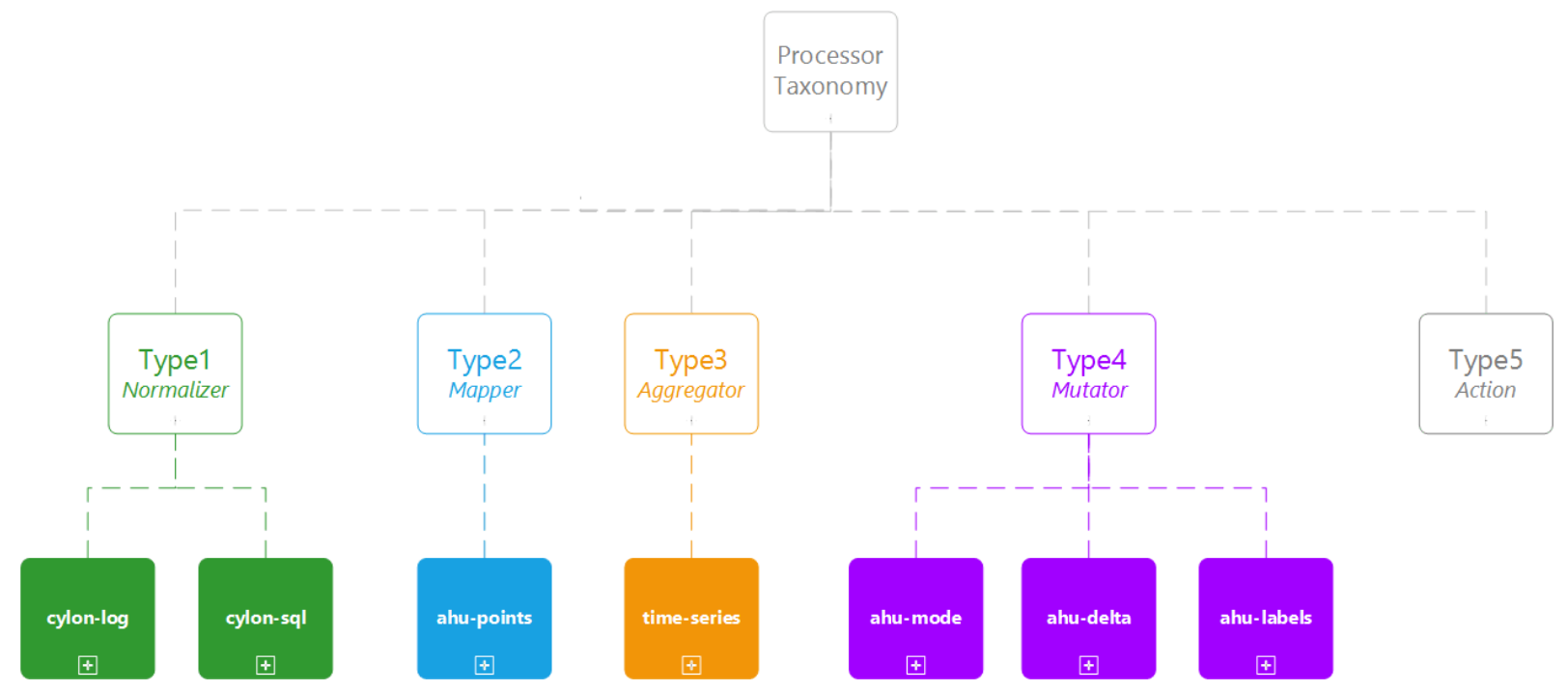

Figure 10. Taxonomy of processing modules in workflow 
Figure 11 shows the native form of BMS energy data transmitted to the data lake. This format is not analyticsready given peculiarities in its structure. Firstly, header information on the first row provides metadata about the log file, such as the measurement type and interval (i.e. 900 seconds), rather than variables or features. Secondly, the structure is primarily designed to provide compact data redundancy for operational data, with each row containing data for the previous 10 days. Column $A$ contains timestamps for the first measurement of each row, which is contained in Column C. For example, the first value on the second row has a timestamp of 14/04/12 17:30, with a corresponding measurement of 23.02. Timestamps for each measurement after Column $C$ must be manually derived by incrementally adding 15-minute values to the first timestamp (i.e. from Column A). Column $B$ specifies the number of measurements on each row, starting from Column $C$ (i.e. first value). Although 1024 measurements are specified for each row in the screenshot, logging issues or outages can affect the number of measurements archived. Given a measurement frequency of 900 seconds (i.e. 15 minutes), each row of 1024 measurements should contain 10 days of data, which means adjacent rows overlap with 9 days of redundant data. Each row in the screenshot shows data logging at the same time every 24 hours (i.e. 5:30pm), but it was common for this pattern to shift without warning, which meant several control checks were needed to ensure timestamps and measurements were parsed correctly.

\begin{tabular}{|c|c|c|c|c|c|c|c|}
\hline 1 & A & B & c & D & $E$ & $\mathbf{F}$ & G \\
\hline 1 & UC32net - 004 Free Cooling & UC3216-008-AHU9 & $\pi$ T-509-901 & 900 & & & \\
\hline 2 & $14 / 04 / 1217: 30$ & 1024 & 23.02 & 22.7 & 22.76 & 22.7 & 22.36 \\
\hline 3 & $15 / 04 / 1217: 30$ & 1024 & 22.29 & 22.33 & 22.16 & 21.63 & 21.33 \\
\hline 4 & $16 / 04 / 1217: 30$ & 1024 & 23.18 & 22.89 & 22.64 & 22.05 & 21.44 \\
\hline 5 & $17 / 04 / 1217: 30$ & 1024 & 21.82 & 21.87 & 21.78 & 21.65 & 21.71 \\
\hline 6 & $18 / 04 / 1217: 30$ & 1024 & 22.62 & 22.67 & 22.63 & 22.52 & 22.6 \\
\hline 7 & $19 / 04 / 1217: 30$ & 1024 & 23.93 & 23.83 & 23.65 & 23.48 & 23.46 \\
\hline 8 & $20 / 04 / 1217: 30$ & 1024 & 23.11 & 23.2 & 23.19 & 23.08 & 23.25 \\
\hline 9 & $21 / 04 / 1217: 30$ & 1024 & 23.28 & 23.49 & 23.41 & 23.33 & 23.35 \\
\hline 10 & $22 / 04 / 1217: 30$ & 1024 & 21.63 & 21.6 & 21.5 & 21.82 & 21.45 \\
\hline 11 & $23 / 04 / 1217: 30$ & 1024 & 21.45 & 21.52 & 21.44 & 21.81 & 21.69 \\
\hline 12 & $24 / 04 / 1217: 30$ & 1024 & 22.6 & 22.61 & 22.77 & 22.62 & 22.73 \\
\hline 13 & $25 / 04 / 1217: 30$ & 1024 & 22.35 & 22.21 & 22.33 & 22.17 & 22.05 \\
\hline 14 & $26 / 04 / 1217: 30$ & 1024 & 21.54 & 21.72 & 21.77 & 21.78 & 21.98 \\
\hline 15 & $27 / 04 / 1217: 30$ & 1024 & 22.87 & 22.75 & 22.7 & 22.73 & 22.7 \\
\hline 16 & $28 / 04 / 1217: 30$ & 1024 & 21.93 & 21.8 & 21.6 & 21.48 & 21.43 \\
\hline
\end{tabular}

Figure 11. Ingested $\log$ file for AHU return air temperature

The first two stages in the AHU workflow transformed the Cylon BMS format to a basic time-series. Figure 12 shows the data output after cylon-log (stage 1 processing module) and ahu-points (stage 2 processing module) were applied. This shows data redundancy has been removed, with each row associated with a single observation (i.e. point-in-time), and each column representing a single measurement. The normalization of BMS data provided subsequent processing modules with a more conventional format upon which to execute data transformations.

\begin{tabular}{c|c|r|}
\hline & \multicolumn{1}{c|}{ A } & \multicolumn{1}{c|}{ B } \\
\hline 1 & Timestamp & \multicolumn{1}{|c|}{ Value } \\
\hline 2 & $14 / 04 / 1217: 30$ & 23.02 \\
\hline 3 & $14 / 04 / 1217: 45$ & 22.7 \\
\hline 4 & $14 / 04 / 1218: 00$ & 22.76 \\
\hline 5 & $14 / 04 / 1218: 15$ & 22.7 \\
\hline 6 & $14 / 04 / 1218: 30$ & 22.36 \\
\hline 7 & $14 / 04 / 1218: 45$ & 22.43 \\
\hline 8 & $14 / 04 / 1219: 00$ & 22.26 \\
\hline 9 & $14 / 04 / 1219: 15$ & 21.99 \\
\hline 10 & $14 / 04 / 1219: 30$ & 21.9 \\
\hline
\end{tabular}

Figure 12. AHU return air temperature after cylon-log

Figure 13 shows the output from stage 3, where individual sensor logs for AHU9 were merged to a tidy dataset. This dataset represents a single entity (i.e. AHU9), with each row containing a single observation (i.e. point-in-time), and each column containing a single measurement (e.g. return air temperature). The availability of such formats can greatly reduce the data wrangling and pre-processing effort associated with data analytics. Table 5 provides a summary of the AHU naming convention used to label columns.

\begin{tabular}{|c|c|c|c|c|c|c|c|c|}
\hline$=$ & $\mathbf{A}$ & B & C & D & $\mathbf{E}$ & $\mathbf{F}$ & G & $\mathbf{H}$ \\
\hline 1 & Timestamp & retT & outT & $\operatorname{mix} T$ & heat & cooT & supT & zonT \\
\hline 2 & $14 / 04 / 1200: 00$ & 21.11 & 0 & 7.7 & 7.55 & 7.62 & 11.72 & 20.72 \\
\hline 3 & $14 / 04 / 1200: 15$ & 21.53 & 0 & 7.46 & 7.11 & 7.15 & 11.23 & 20.82 \\
\hline 4 & $14 / 04 / 12$ 00:30 & 21.78 & 0 & 8.83 & 8.37 & 8.52 & 12 & 20.93 \\
\hline 5 & $14 / 04 / 1200: 45$ & 21.97 & 0 & 9.07 & 8.54 & 8.63 & 12.03 & 21.07 \\
\hline 6 & $14 / 04 / 12$ 01:00 & 22 & 0 & 8.83 & 8.28 & 8.49 & 11.94 & 21.14 \\
\hline 7 & $14 / 04 / 12$ 01:15 & 22.07 & 0 & 9.14 & 8.7 & 8.8 & 12.15 & 21.23 \\
\hline 8 & $14 / 04 / 12$ 01:30 & 22.2 & 0 & 8.9 & 8.39 & 8.53 & 11.79 & 21.25 \\
\hline 9 & $14 / 04 / 12$ 01:45 & 22.14 & 0 & 9.03 & 8.69 & 8.9 & 12.13 & 21.21 \\
\hline 10 & $14 / 04 / 120$ & 22.24 & 0 & 9.21 & 8.59 & 8.63 & 12.1 & 21.29 \\
\hline 11 & $14 / 04 / 12$ 02:15 & 22.29 & 0 & 8.68 & 8.14 & 8.39 & 11.72 & 21.36 \\
\hline 12 & $14 / 04 / 12 \mathrm{C}$ & 22.07 & 0 & 8.98 & 8.5 & 8.66 & 12.11 & 1.38 \\
\hline 13 & $14 / 04 / 12$ 02:45 & 22.1 & 0 & 8.73 & 8.17 & 8.42 & 11.93 & 21.39 \\
\hline 14 & $14 / C$ & 22.15 & 0 & 8.82 & 18 & 8.34 & 12.06 & 1.38 \\
\hline 15 & $14 / C$ & 22.35 & v & 8.68 & 8.13 & 8.28 & 11.91 & 21.41 \\
\hline 16 & $14 / 04 / 120$ & 22.49 & 0 & 8.61 & 8.03 & 8.28 & 11.85 & 21.44 \\
\hline 17 & $14 / 04 / 12 \mathrm{C}$ & 22.52 & 0 & 8.6 & 8.2 & 8.29 & 12.04 & 21.48 \\
\hline 18 & $14 / 04 / 120$ & 22.28 & 0 & 8.4 & 8.04 & 8.19 & 11.9 & 21.43 \\
\hline 19 & $14 / 04 / 120$ & 22.11 & 0 & 8.9 & 8.6 & 8.56 & 12.03 & 21.38 \\
\hline 20 & $14 / 04 / 120$ & 22.03 & 0 & 8.69 & 8.47 & 8.56 & 12.01 & 21.37 \\
\hline 21 & $14 / 04 / 120$ & 21.84 & 0 & 8.47 & 8.14 & 8.25 & 11.71 & 21.26 \\
\hline 22 & $14 / 04 / 120$ & 21.79 & 0 & 8.81 & 8.5 & 8.48 & 12.1 & 21.21 \\
\hline 23 & $14 / 04 / 120$ & 22.06 & 0 & 14.98 & 11.08 & 9.24 & 12.62 & 21.41 \\
\hline 24 & $14 / 04 / 120$ & 21.53 & 0 & 16.4 & 12.39 & 9.7 & 14.3 & 21.64 \\
\hline 25 & $14 / 04 / 12$ 05:45 & 21.09 & 0 & 17.03 & 13.31 & 10.42 & 15.21 & 21.85 \\
\hline 26 & $14 / 04 / 12$ 06:00 & 20.93 & 0 & 16.93 & 13.93 & 11.37 & 15.77 & 22.01 \\
\hline 27 & $14 / 04 / 1206: 15$ & 20.89 & 0 & 16.35 & 14.07 & 12.09 & 16.29 & 22.06 \\
\hline
\end{tabular}

Figure 13. AHU log file after time-series merge 


\begin{tabular}{|c|c|c|}
\hline Section & Measurement & Convention \\
\hline Return air & Temperature $\left[{ }^{\circ} \mathrm{C}\right]$ & $\operatorname{ret} \mathrm{T}$ \\
\hline Return air & Humidity [\%] & retH \\
\hline Return air & Enthalpy $[\mathrm{kJ} / \mathrm{kg}]$ & retE \\
\hline Return air & $\mathrm{CO} 2[\mathrm{ppm}]$ & retC \\
\hline Return air & VSD [\%] & retVSD \\
\hline Return air & Flow $[\mathrm{m} 3 / \mathrm{s}]$ & retF \\
\hline Return air & Motor power $[\mathrm{kW}]$ & retM \\
\hline Return air & Damp. Position [\%op] & $\operatorname{detD}$ \\
\hline Exhaust air & Damp. Position [\%op] & exhD \\
\hline Outside air & Temperature $\left[{ }^{\circ} \mathrm{C}\right]$ & out \\
\hline Outside air & Temperature $2\left[{ }^{\circ} \mathrm{C}\right]$ & outT2 \\
\hline Outside air & Humidity [\%] & outH \\
\hline Outside air & Humidity $2[\%]$ & outH2 \\
\hline Outside air & Enthalpy $[\mathrm{kJ} / \mathrm{kg}]$ & outE \\
\hline Outside air & Enthalpy $2[\mathrm{~kJ} / \mathrm{kg}]$ & outE2 \\
\hline Outside air & Damp. Position [\%op] & outD \\
\hline Frost coil & Supply water $\left[{ }^{\circ} \mathrm{C}\right]$ & froS \\
\hline Frost coil & Return water $\left[{ }^{\circ} \mathrm{C}\right]$ & froR \\
\hline Frost coil & Valve position [\%op] & froV \\
\hline Frost coil & Off coil air $\left[{ }^{\circ} \mathrm{C}\right]$ & froT \\
\hline Mixed air & Temperature $\left[{ }^{\circ} \mathrm{C}\right]$ & $\operatorname{mixT}$ \\
\hline Mixed air & Humidity [\%] & $\operatorname{mixH}$ \\
\hline Mixed air & Enthalpy $[\mathrm{J} / \mathrm{kg}]$ & $\operatorname{mixE}$ \\
\hline Heating coil & Supply water $\left[{ }^{\circ} \mathrm{C}\right]$ & heaS \\
\hline Heating coil & Return water $\left[{ }^{\circ} \mathrm{C}\right]$ & hear \\
\hline Heating coil & Valve position.[\%op] & heaV \\
\hline Heating coil & Off coil air $\left[{ }^{\circ} \mathrm{C}\right]$ & heaT \\
\hline Cooling coil & Supply water $\left[{ }^{\circ} \mathrm{C}\right]$ & $\operatorname{cooS}$ \\
\hline Cooling coil & Return water $\left[{ }^{\circ} \mathrm{C}\right]$ & cooR \\
\hline Cooling coil & Valve position.[\%op] & $\mathrm{cooV}$ \\
\hline Cooling coil & Off coil air $\left[{ }^{\circ} \mathrm{C}\right]$ & $\operatorname{cooT}$ \\
\hline Humidification & Temperature $\left[{ }^{\circ} \mathrm{C}\right]$ & humT \\
\hline Humidification & Humidity [\%] & humH \\
\hline Humidification & Dew-point $\left[{ }^{\circ} \mathrm{C}\right]$ & humDew \\
\hline Humidification & Status [1/0] & humSta \\
\hline Humidification & Valve position. [\%op] & humV \\
\hline Reheat coil & Supply water $\left[{ }^{\circ} \mathrm{C}\right]$ & rehS \\
\hline Reheat coil & Return water $\left[{ }^{\circ} \mathrm{C}\right]$ & rehR \\
\hline Reheat coil & Valve position. [\%op] & rehV \\
\hline Reheat coil & Off coil air temperature $\left[{ }^{\circ} \mathrm{C}\right]$ & rehT \\
\hline Supply air & Temperature $\left[{ }^{\circ} \mathrm{C}\right]$ & supT \\
\hline Supply air & Humidity [\%] & supH \\
\hline Supply air & Enthalpy $[\mathrm{kJ} / \mathrm{kg}]$ & supE \\
\hline
\end{tabular}

\begin{tabular}{l|l|l}
\hline Supply air & CO2 [ppm] & supC \\
\hline Supply air & VSD $[\%]$ & supVSD \\
\hline Supply air & Flow $[\mathrm{m} 3 / \mathrm{s}]$ & supF \\
\hline Supply air & Motor power $[\mathrm{kW}]$ & supM \\
\hline Supply air & Pressure $[\mathrm{Pa}]$ & Sup \\
\hline Zone & Temperature $\left[{ }^{\circ} \mathrm{C}\right]$ & zonT \\
\hline Zone & Humidity $[\%]$ & zonH \\
\hline Zone & $\mathrm{CO} 2[\mathrm{ppm}]$ & zonC \\
\hline
\end{tabular}

Table 5. AHU conventions (Bruton et al., 2014)

Figure 14 shows new variables appended to the AHU dataset after the execution of ahu-mode and ahu-delta processing modules. The mode variable refers to the current operating mode of the AHU. These operating modes are classified in Table 5, while Figure 15 illustrates the logical transition from heating to cooling modes. The appended delta variables refer to temperature differentials between the off-coil heating (heaT) and cooling (cooT) temperatures, and the units mixing box (mixT). While mode may indicate the system-level state (e.g. heating or cooling), deltaCooT and deltaHeaT can infer component-level state. Comparing these measurements for state consistency may indicate potential operating issues.

\begin{tabular}{|r|r|r|r|r|r|}
\multicolumn{1}{|c|}{$\mathbf{P}$} & \multicolumn{1}{|c|}{$\mathbf{Q}$} & \multicolumn{1}{|c|}{$\mathbf{R}$} & \multicolumn{1}{|c|}{$\mathbf{S}$} & \multicolumn{1}{c|}{$\mathbf{U}$} \\
\hline supF & supVSD & retVSD & mode & deltaCooT & deltaHeaT \\
\hline 19.98 & 36.24 & 60.38 & 3 & -3.38 & 0.06 \\
\hline 19.98 & 36.82 & 62 & 3 & -2.21 & 0.1 \\
\hline 20 & 36.71 & 62.66 & 3 & 1.44 & -0.03 \\
\hline 19.97 & 37.46 & 63.52 & 3 & 0.67 & 0.04 \\
\hline 19.99 & 37.52 & 64.11 & 3 & -0.48 & 0.06 \\
\hline 20.01 & 36.96 & 64.29 & 3 & 1.43 & 0.06 \\
\hline 19.96 & 37.8 & 64.49 & 3 & 2.7 & 0.04 \\
\hline 19.97 & 38.08 & 64.88 & 3 & 1.33 & 0.15 \\
\hline 19.99 & 37.88 & 65.03 & 3 & -0.12 & 0.13 \\
\hline 20 & 37.55 & 64.79 & 3 & 2.02 & 0.1 \\
\hline 19.97 & 38.05 & 64.95 & 3 & 1.18 & 0.01 \\
\hline 20.03 & 37.44 & 64.66 & 3 & 0.69 & 0.1 \\
\hline 19.98 & 37.4 & 64.29 & 2 & 3.42 & 0.44 \\
\hline 20.02 & 37.6 & 64.3 & 3 & 2.5 & 0.05 \\
\hline 20.03 & 37.3 & 64.31 & 3 & 1.38 & 0.08 \\
\hline 19.99 & 37.67 & 64.22 & 3 & 0.92 & 0.06 \\
\hline
\end{tabular}

Figure 14. AHU log file after ahu-mode and ahu-delta 


\begin{tabular}{l|l}
\hline Name & Description \\
\hline Mode 1 & Heating with Minimum Outside Air \\
\hline Mode 2 & $\begin{array}{l}\text { Modulation of Fresh air with Return } \\
\text { air with no heating or cooling }\end{array}$ \\
\hline Mode 3 & Maximum outside air with Cooling \\
\hline Mode 4 & Minimum outside air with Cooling
\end{tabular}

Table 6. AHU modes of operation

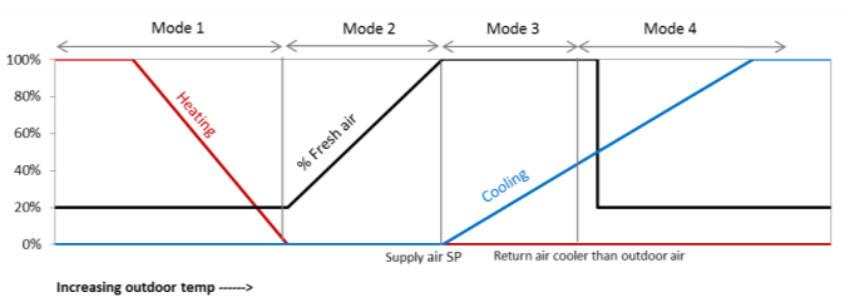

Figure 15. AHU control sequence and modes

The data processing logic for deriving operating modes, offcoil delta temperatures and diagnostic classifications are based on engineering first principles presented in previous research (Bruton et al., 2014).

\subsection{Step 5 - Model Building}

The AHU workflow output an analytics-ready dataset to support the development of a data-driven model. This dataset was used to profile heating and cooling operations in the unit, develop a Support Vector Machine (SVM) (Jedliński \& Jonak, 2015) for heating coil issues, and create a deployable model using PMML. The RStudio integrated development environment for statistical analysis, modelling and visualization, was used for analytics activities. These activities leveraged several $R$ packages, including GGPlot2 and base R library for time-series visualization, TidyR for data wrangling, Dplyr for data manipulation, E1071 for building an SVM model, and PMML for encoding the model, while data acquisition from the AHU workflow was implemented using HTTP GET requests, with site, workflow and stage parameters used to identify the dataset.

The process began with an initial data exploration of the AHU's operating trends. Figure 16 profiles deltaHeaT (red) and deltaCooT (blue) measurements from the AHU dataset. These measurements show temperature differences between off-coil heating and cooling sensors, and the units mixing box, over a 4 year period. The analysis showed the unit predominantly focused on cooling, with a consistent cycle visible throughout the time-series. In contrast, heating patterns showed inconsistent and sporadic spikes, with some persistent heating visible $75 \%$ of the way through the timeseries. While sudden surges of heating or cooling may be caused by control/mode changes, consistently high/low readings may indicate an underlying issue.
A couple of observations were identified in the delta temperature profiles for further investigation. First, we investigated the AHU's usage in the facility due to the strong cooling pattern. Given Ireland's moderate climate such patterns would not be expected. However, after presenting this pattern to operational staff, we discovered the unit services the factory floor. Therefore, given the residual heat generated from equipment, the unit (i.e. AHU9) is continually cooling the space. Second, the delta heating pattern was unaffected when there was strong cooling, but cooling patterns were affected by heating surges. After discussions with maintenance personnel, we discovered this pattern occurred due to the heating sensor being positioned before the cooling sensor (i.e. air is cooled after passing the heating sensor). Third, given the AHU predominantly operates in cooling mode, identifying issues with cooling components may be difficult.

\section{deltaHeaT deltaCooT}

\section{Delta Temperature Profile for AHU9}

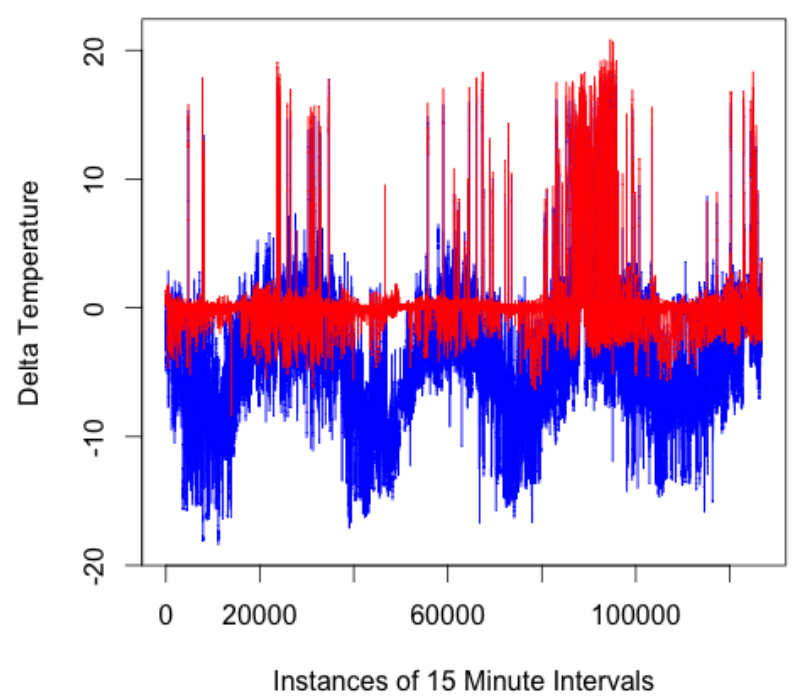

Figure 16. Delta temperature off-coil heating and cooling

Correlation analysis was undertaken to identify relationships in the AHU dataset, with the intention of informing feature selection, which was predominantly based on the subject matter expertise of the researchers. A correlation describes the relationship between two measurements, which may be either positive or negative. Positive correlations occur when one variable increases or decreases, in response to an increase or decrease in the other. Negative correlations occur when one variable increases, in response to a decrease in the other, or vice versa.

Figure 17 shows the correlation matrix created from the AHU dataset. The relationships of interest were those which correlated with heating and cooling. Without considering special circumstances, engineering first principles indicate 
correlations should exist between outside temperature (outT), heating (deltaHeaT) and cooling (deltaCooT). While outside temperature (outT) was correlated with deltaCooT, it had no correlation with deltaHeaT. In this instance, the poor correlation between outside temperature and heating can be attributed to the AHU's role, which is to continually cool the production space, meaning heating components are less likely to be engaged in response to outside temperature changes. Several other negative and positive correlations were associated with deltaCooT, all of which were assessed and validated using subject matter expertise.

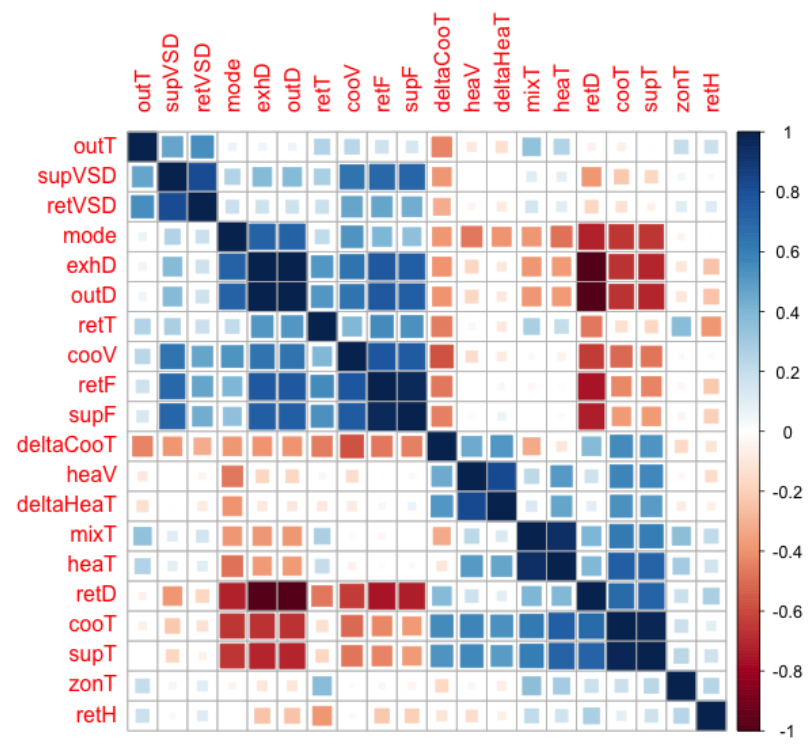

Figure 17. AHU measurement correlation matrix

Given the strong correlation between outside air temperature (outT) and off-coil cooling delta temperature (deltaCooT), time-series analysis was used to visualize how cooling patterns modulated in response to outside temperature. Given this analysis focused on the visualization of the units cooling patterns, measurements associated with AHU mode 1 (i.e. heating) were removed. Figure 18 shows outT (green) and deltaCooT (blue) measurements in a time-series. The pattern shows increases in outside temperature trigger the unit to increase cooling. Similarly, decreases in outside temperature trigger the unit to decrease cooling. This system-level health check of the AHU's cooling operation did not identify any obvious issues.

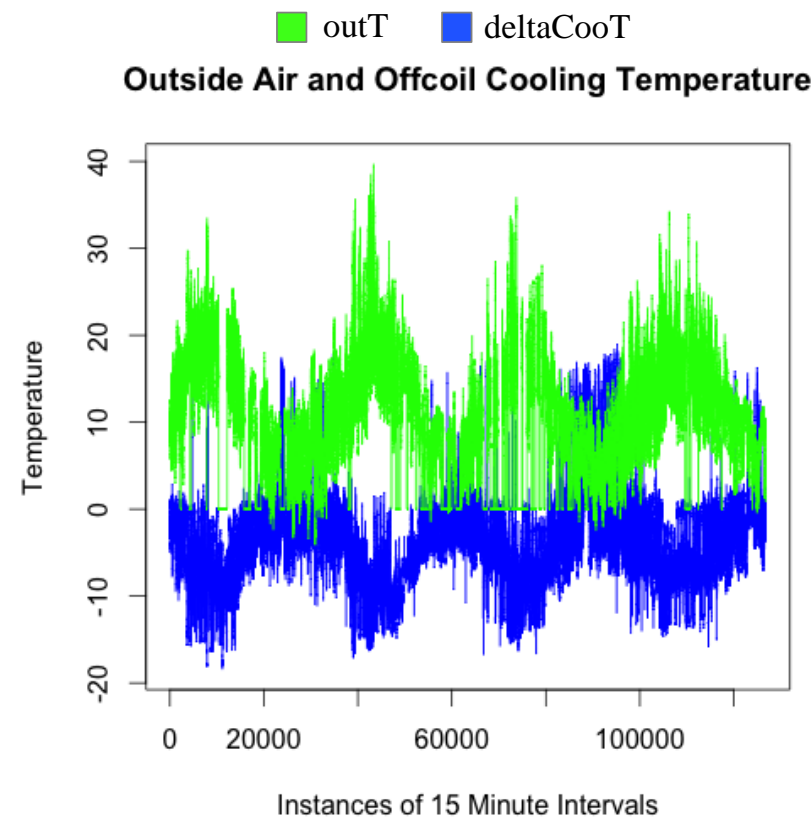

Figure 18. Outside air and off-coil cooling delta temperature

The original delta temperature profile in Figure 16 displayed unusual heating behavior $75 \%$ of the way through the timeseries. Based on existing knowledge and subject matter expertise, AHU's that are in heating mode (i.e. mode 1), and have a delta heating measurement of one degree or more, may be indicative of component-level issues. Therefore, the previously identified heating surge warranted further investigation. Before additional analysis was undertaken, measurements recorded while the unit was in heating mode (i.e. mode 1) were removed, so any heating surges could be considered a conflict with the AHU's system-level mode. Figure 19 illustrates the time-series for deltaHeaT with a 10 day rolling average applied to reduce noise. Similar to the original temperature profile, there is a sustained surge in heating at $75-80 \%$ of the way through the time-series. Error thresholds of plus and minus one degree were added to visualize normal operation of the heating component. Given the existence of data characterizing a heating component issue, the next step was to train and encode a data-driven model, which could later be deployed in the factory to identify these issues in real-time. 
deltaHeaT

\section{Offcoil Heating Temperature with Error Threshold}

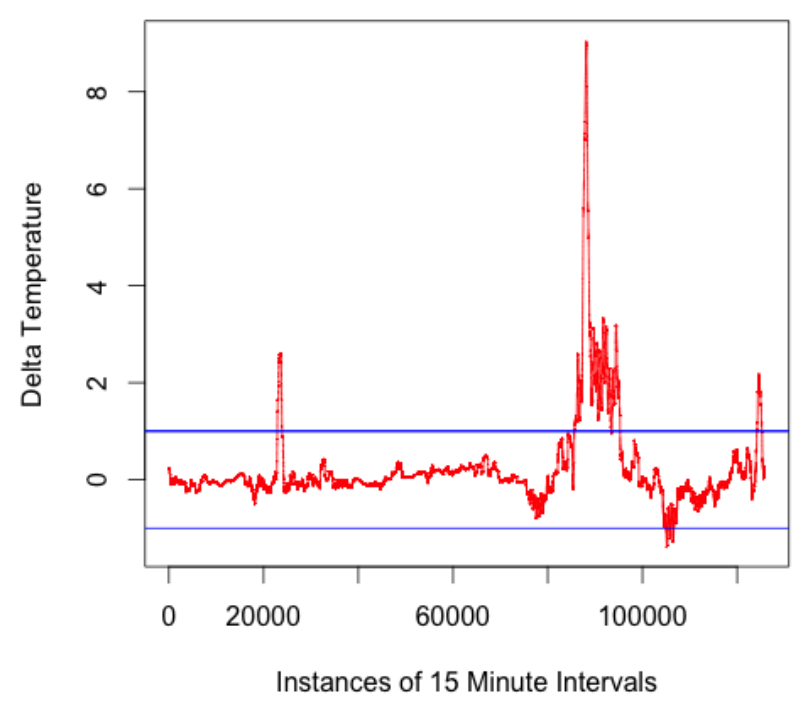

Figure 19. Delta off-coil heating 10 day moving average

The original dataset of AHU measurements contained 126,487 records at 15 minute resolution. Given most measurements were not examples of heating component issues, a random filtering process was applied to produce 18,979 training examples, and 8,135 test examples. Each of these datasets contained a 70/30 split of examples with and without heating component issues. This redistribution was applied to increase the strength of the issue signal, with the expectation that relevant training data would improve the prediction performance of the model. Given the prior experience and skillset of the authors, a Support Vector Machine (SVM) was chosen to build a binary classification model for heating component issue identification, which was implemented using the E1071 package in $\mathrm{R}$. To reduce the hypothesis search space, dimensionality reduction was applied to training and test datasets using engineering first principles, with some verification and validation from the correlation matrix. This resulted in the final training and test datasets consisting solely of temperature measurements, which was consistent with other analysis undertaken during this study (e.g. using delta temperature measurements for assessing AHU operation).

Figure 21 shows a screenshot of the confusion matrix generated for the SVM model. This conveys the predictive performance of the model, which was determined using the available test data. The model predicted there were no issues with 6,019 of the examples. Of these predictions, it correctly asserted no issue 5,927 times, and incorrectly asserted no issue 92 times. Therefore, the model correctly predicted there were no heating issues in $98 \%$ of examples. In addition, the model predicted there were issues in 2,116 of examples. These predictions were correct for 2,043 examples, and incorrect for 73 examples, which means the model correctly predicted a heating issue in $97 \%$ of examples. The high prediction accuracy may be attributed to large quantities of training data containing examples of the heating issue, while the concept to be learned was simplified by (a) restricting features to temperature measurements, and (b) limiting prediction to binary classification.

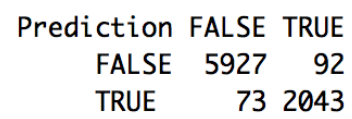

Figure 20. SVM model performance confusion matrix

Operationalizing data-driven models in real-time enterprise environments traditionally requires models to be interpreted and coded using an imperative programming language, such as $\mathrm{C}++$ or Java. However, PMML is an emerging XMLbased standard that describes predictive models, which may be interpreted and executed using compliant predictive scoring engines. This facilitates model development using different statistical tools and applications, while also ensuring these models are accessible to embedded applications in the factory. Figure 21 shows the heating component issue identification SVM model encoded as PMML. This markup was generated using the PMML package in $\mathrm{R}$, which supports the automatic encoding of models built using the E1071 package. To make this model accessible to applications in the factory, the PMML file was published to S3 and assigned a unique URL.

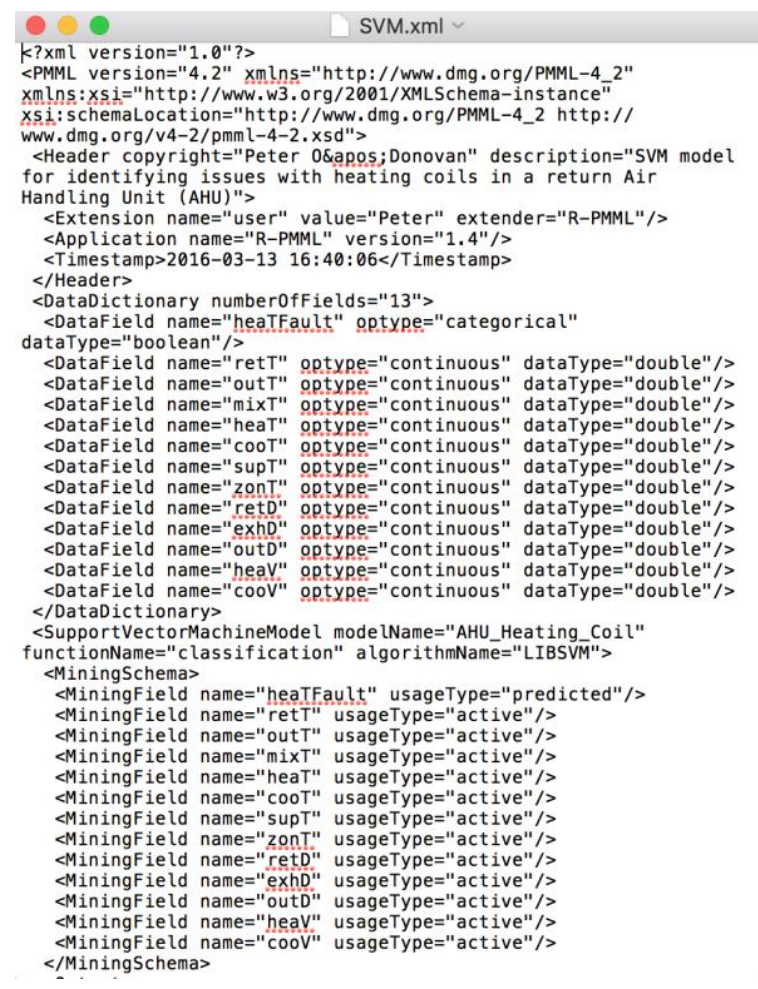

Figure 21. PMML encoded predictive model 


\subsection{Step 6 - Operationalize Model}

To enable real-time monitoring of heating components, a PMML compliant predictive scoring engine was setup on the cloud. This served as an endpoint for embedded applications in the factory to transmit real-time operational data using web services, and receive notifications of heating component issues. The scoring engine used during this study was OpenScoring (GitHub, 2016), which is a Javabased engine available under the GNU Affero General Public License (APGL). This engine was deployed on an Amazon EC2 compute instance, and configured to use the PMML-encoded SVM model residing on S3.

Figure 22 illustrates the sequential interactions of the realtime monitoring process. First, an embedded application on a networked computer polls the relevant PLC to retrieve the AHU's current operating measurements. Second, these measurements are transmitted to the OpenScoring web service to serve as input for the issue identification model. Third, the scoring engine retrieves the PMML-encoded SVM model from S3, and predicts whether an issue exists from the input data provided. Finally, the scoring engine returns a result to the embedded application with zero-ormore issues (i.e. empty array indicates no issues). These interactions could be extended to include other systems and processes, such as the propagation of identified faults to maintenance and health monitoring systems, but these were not of primary importance to this study.

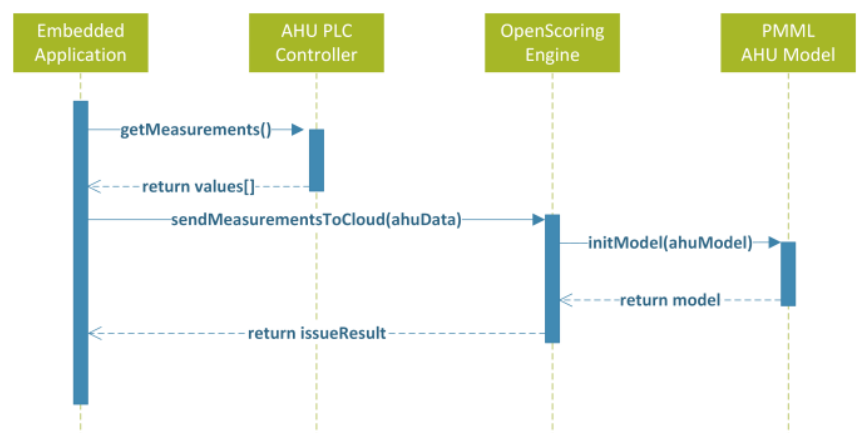

Figure 22 Sequence diagram for embedded operations

Given the exploratory nature of this research, an OPC simulator (MatrikonOPC, 2016) was used as a surrogate for production PLC's to avoid any potential interference with factory operations. The simulator was deployed on the BMS $\mathrm{PC}$ and configured to use the AHU conventions presented in Table 5. This simulator was polled at 60 second intervals by a C\# .NET background application to acquire current operating measurements, which were transmitted to the OpenScoring engine for evaluation. Where the scoring engine response included an identified issue, the background application recorded the timestamp and all sensor measurements for that point in time, as well as the identified issue (i.e. heating component). The background application was programmed to terminate after logging 20 issues to enable engineers on-site to manually evaluate the predictions. This manual evaluation process was undertaken over a 7 day period, where 140 predictions were logged by the background application, with $100 \%$ of these predictions determined as correct, based on engineering first principles relating to AHU diagnostics (Bruton et al., 2014).

\section{DISCUSSION}

The following section discusses findings from the case study. These findings are discussed in the context of the roles and responsibilities from the industrial analytics methodology. These roles are (1) Data Integration and Management, and (2) Model Building and Deployment.

\subsection{Data Integration and Management}

Data ingestion was implemented and tested on two different computing environments. In both cases, ingesting the BMS archive of $838 \log$ files, with up to 4 years of data, took between 10 and 39 minutes. Differences in these execution times were broadly attributed to differing hardware specifications (e.g. solid state drive and greater processing power), active background processes, and available upload bandwidth. However, these findings demonstrated the impact technologies and infrastructure may have on factoryto-cloud data integration.

Enterprise development technologies (e.g. C\# .NET, J2EE etc.) were used for data ingestion. Given the adequate performance of the ingestion process, there does not appear to be an immediate need to investigate other tools, such as those associated with Big Data. Results indicated hardware specification and bandwidth impacted ingestion execution time, with a mid-range development PC completing the process three times faster than an older BMS PC. However, as facilities progress towards smart manufacturing, an increase in sensing technologies, coupled with an increase in measurement resolution, will inevitably place more stress on ingestion processes. To address these scaling and performance challenges, ingestion processes may (a) horizontally scale by deploying processes across multiple computers, (b) vertically scale by increasing hardware specification, (c) increase upload bandwidth capacity, or (d) optimize multithreading to leverage parallelism.

The energy data collected from the factory highlighted potential peculiarities in proprietary data sources. Such data is not analytics-ready and requires complex processing to present it in a useful form. While ad hoc scripts could have been used to reshape the energy data, without formal processes to create, share and reuse these workflows, there is an inherent risk of duplicating time-consuming data processing tasks. Given data cleaning and transformation consumes much of the effort in analytics projects, while providing the lowest immediate value, facilities developing industrial analytics capabilities for smart manufacturing 
should develop formal methods and architectures to standardize and automate these processes.

The implementation of a formal multistage workflow for preparing industrial time-series data was presented in this research. This workflow used scalable and interchangeable cloud-based data processing modules to transform AHU data to an analytics-ready state. In addition, the multistage aspect of the workflow demonstrated how exposing outputs at each processing stage may facilitate reusability, while potentially reducing duplicated effort. Such an approach contrasts with input/output workflows, whereby stepwise transformations are encapsulated and inaccessible postexecution. To illustrate the reuse potential of the multistage workflow, consider a scenario where a workflow is designed to output AHU data as a daily average for an analytics project. In the future, another analytics project needs access to the same data but at its original resolution (e.g. 15 minute intervals). Where outputs from multistage workflows are accessible, the original data may be acquired from an earlier stage in the existing workflow, without undertaking additional data integration and cleaning.

\subsection{Model Building and Deployment}

Turnkey analytics was demonstrated using a simple issue identification scenario for AHU heating components. Data exploration and modeling activities were undertaken using RStudio, with HTTP used to acquire analytics-ready data from the AHU workflow, which served to reduce common data processing overheads. Where similar data management and governance strategies do not facilitate seamless analytics pipelines, data processing overheads are likely to impede analytics outputs and productivity. The analyticsready AHU data from the workflow was used to train an SVM model to identify heating component issues, and encoded using XML-based PMML markup to promote interoperability and enable model deployment. While discussions regarding standards are prevalent in Operation and Information Technology, this research also highlights the importance and usefulness of open non-proprietary standards in analytics pipelines and processes.

Operationalizing and embedding analytics is central to smart manufacturing. While data analytics and business intelligence activities can derive useful insights, their true impact may not be known until they can affect real-time decision-making in the factory. Therefore, facilities should appreciate the differences between model development and deployment, and data architectures that support both use cases (i.e. industrial analytics lifecycle). Model deployment in this research used a cloud-based scoring engine, which linked to the previous created PMML model. This real-time deployment was demonstrated using a purpose-built background application, which continuously acquired AHU measurements from an OPC simulator, and collaborated with the PMML compliant scoring engine to determine the
AHU state. The issues identified by the embedded analytics application over a 7 day period were manually evaluated by engineers to ensure the technical integrity of the implementation, comprising real-time factory-to-cloud data exchange, and execution of the issue identification predictive model.

\section{Conclusions}

Data-driven operations for smart manufacturing are highly dependent on the availability of industrial analytics capabilities. However, developing these capabilities can be challenging due to the lack of formal and systematic approaches that inform their development. While ad hoc strategies can deliver some benefits, they may also exhibit duplicated effort and inefficient execution, resulting in less impact on operational performance. Industrial analytics is a multi-faceted topic that encompasses aspects of operation technology, information technology, statistics, engineering, and informatics. Given the multidisciplinary nature of industrial analytics, coupled with the diverse technologies and standards that may exist from factory-to-factory, highly prescriptive industrial analytics methodologies and frameworks are not plausible. Instead, facilities should use formal methodologies to support the development of industrial analytics lifecycles, which facilitate the operationalization of analytics models in the factory, while using technologies and standards aligned with their resources and environment.

Thus, an industrial analytics methodology for developing industrial analytics capabilities was presented in this research. The methodology provides formal heuristics for developing teams, architectures and processes that form an industrial analytics lifecycle, while illustrating the technology convergences that occur. This methodology was demonstrated and validated using a case study focusing on a real-world issue identification scenario. The findings and observations compiled during the study highlighted several important points. Firstly, while analytics models deliver operational insights, robust and scalable information and data architectures are needed to support their development and deployment. Secondly, given the technology and analytics convergences that occur in the industrial analytics lifecycle, there is a significant need to adopt standards that facilitate interoperability and integration. Finally, industrial analytics can be delineated from traditional data analytics by the emphasis placed on affecting real-time decision-making in the factory. Where analytics outputs are not embedded in factory operations, the return on investment from analytics initiatives may be diminished.

\section{ACKNOWLEDGEMENT}

The authors would like to thank the Irish Research Council, DePuy Ireland for their funding of this research as part of the Enterprise Partnership Scheme (EPSPG/2013/578). 


\section{REFERENCES}

Al-jaroodi, J., \& Mohamed, N. (2012). Journal of Network and Computer Applications Service-oriented middleware: A survey. Journal of Network and Computer Applications, 35(1), 211-220. doi:10.1016/j.jnca.2011.07.013

Alves Santos, R., Normey-Rico, J. E., Merino Gómez, A., Acebes Arconada, L. F., \& de Prada Moraga, C. (2005). OPC based distributed real time simulation of complex continuous processes. Simulation Modelling Practice and Theory, 13(7), 525-549. doi:10.1016/j.simpat.2005.01.005

Bagheri, B., Yang, S., Kao, H., \& Lee, J. (2015). Cyberphysical Systems Architecture for Self-Aware Machines in Industry 4. 0 Environment. IFAC PapersOnLine, $1622-1627$. doi:10.1016/j.ifacol.2015.06.318

Brandl, D. (2008). What is ISA-95? Industrial Best Practices of Manufacturing Information Technologies with ISA-95 Models, 1-32.

Bruton, K., Raftery, P., O’Donovan, P., Aughney, N., Keane, M. M., \& O’Sullivan, D. T. J. (2014). Development and alpha testing of a cloud based automated fault detection and diagnosis tool for Air Handling Units. Automation in Construction, 39(0), 70-83. doi:10.1016/j.autcon.2013.12.006

Cardiel, I. A., Gil, R. H., Somolinos, C. C., \& Somolinos, J. C. (2012). Expert Systems with Applications A SCADA oriented middleware for RFID technology. Expert Systems With Applications, 39(12), 1111511124. doi:10.1016/j.eswa.2012.03.045

Chand, S., \& Davis, J. (2010). What is Smart Manufacturing? Time Magazine.

Chungoora, N., Young, R. I., Gunendran, G., Palmer, C., Usman, Z., Anjum, N. a., ... Case, K. (2013). A model-driven ontology approach for manufacturing system interoperability and knowledge sharing. Computers in Industry, 64(4), 392-401. doi:10.1016/j.compind.2013.01.003

Data Mining Group. (2016). PMML 4.3. Data Mining Group. Retrieved from http://dmg.org/pmml/v43/GeneralStructure.html

Davis, J., Edgar, T., Porter, J., Bernaden, J., \& Sarli, M. (2012). Smart manufacturing, manufacturing intelligence and demand-dynamic performance. Computers \& Chemical Engineering, 47, 145-156. doi:10.1016/j.compchemeng.2012.06.037

Dworschak, B., \& Zaiser, H. (2014). Competences for cyber-physical systems in manufacturing - first findings and scenarios. Procedia CIRP, 25, 345-350. doi:10.1016/j.procir.2014.10.048

Emerson, D., Kawamura, H., \& Matthews, W. (2007). Plant-to-business interoperability using the ISA-95 standard.

Fosso Wamba, S., Akter, S., Edwards, A., Chopin, G., \&
Gnanzou, D. (2015). How "big data" can make big impact: Findings from a systematic review and a longitudinal case study. International Journal of Production Economics, 1-13. doi:10.1016/j.ijpe.2014.12.031

Giovannini, A., Aubry, A., Panetto, H., Dassisti, M., \& El Haouzi, H. (2012). Ontology-based system for supporting manufacturing sustainability. Annual Reviews in Control, 36(2), 309-317. doi:10.1016/j.arcontrol.2012.09.012

GitHub. (2016). OpenScoring. Retrieved from https://github.com/jpmml/openscoring

Gligor, A., \& Turc, T. (2012). Development of a Service Oriented SCADA System. Procedia Economics and Finance, 3(12), 256-261. doi:10.1016/S22125671(12)00149-9

Hazen, B. T., Boone, C. a., Ezell, J. D., \& Jones-Farmer, L. A. (2014). Data quality for data science, predictive analytics, and big data in supply chain management: An introduction to the problem and suggestions for research and applications. International Journal of Production Economics, 154, 72-80. doi:10.1016/j.ijpe.2014.04.018

Heng, S. (2014). Industry 4.0: Huge potential for value creation waiting to be tapped. Deutsche Bank Research. Retrieved from http://www.dbresearch.com/servlet/reweb2.ReWEB?r wsite=DBR_INTERNET_EN-

PROD\&rwobj=ReDisplay.Start.class\&document=PR OD0000000000335628

Hong, X., \& Jianhua, W. (2006). Using standard components in automation industry: A study on OPC Specification. Computer Standards \& Interfaces, 28(4), 386-395. doi:10.1016/j.csi.2005.05.001

ISA. (2016a). ISA-88. Retrieved from http://www.isa88.com

ISA. (2016b). ISA-95. Retrieved from http://www.isa95.com

Jedliński, Ł., \& Jonak, J. (2015). Early fault detection in gearboxes based on support vector machines and multilayer perceptron with a continuous wavelet transform. Applied Soft Computing, 30, 636-641. doi:10.1016/j.asoc.2015.02.015

Kastner, W., Neugschwandtner, G., Soucek, S., \& Newman, H. M. (2005). Communication Systems for Building Automation and Control, 93(6).

Kumar, P., Dhruv, B., Rawat, S., \& Rathore, V. S. (2014). Present and future access methodologies of big data. International Journal of Advance Research in Science and Engineering, 8354(3), 541-547.

Lee, J. (2014). Recent Advances and Transformation Direction of PHM. NIST. Retrieved August 3, 2015, from http://www.nist.gov/el/isd/upload/Keynote_Lee_IMSUniversity_of_Cincinnati_updated.pdf

Lee, J., Ardakani, H. D., Yang, S., \& Bagheri, B. (2015). 
Industrial Big Data Analytics and Cyber-physical Systems for Future Maintenance \& Service Innovation. Procedia CIRP, 38, 3-7. doi:10.1016/j.procir.2015.08.026

Lee, J., Bagheri, B., \& Kao, H. (2015). A Cyber-Physical Systems architecture for Industry 4.0-based manufacturing systems. Manufacturing Letters, 3(September 2015), 18-23. doi:10.1016/j.mfglet.2014.12.001

Lee, J., Kao, H.-A. A., \& Yang, S. (2014). Service Innovation and Smart Analytics for Industry 4.0 and Big Data Environment. Procedia CIRP, 16, 3-8. doi:10.1016/j.procir.2014.02.001

Lee, J., Lapira, E., Bagheri, B., \& Kao, H. (2013). Recent advances and trends in predictive manufacturing systems in big data environment. Manufacturing Letters, 1(1), 38-41. doi:10.1016/j.mfglet.2013.09.005

Manufacturing, S., Manufacturing, C. S., Coalition, L., Smart, T., Leadership, M., Incorporated, E., ... Any, D. (2011). Implementing 21st Century Smart Manufacturing.

MatrikonOPC. (2016). MatrikonOPC Simulation Server. Retrieved from https://www.matrikonopc.com/products/opcdrivers/opc-simulation-server.aspx

McKinsey. (2011). Big data: The next frontier for innovation, competition, and productivity.

Nagorny, K., Colombo, A. W., \& Schmidtmann, U. (2012). A service and multi-agent-oriented manufacturing automation architecture. Computers in Industry, 63(8), 813-823. doi:10.1016/j.compind.2012.08.003

O’Donovan, P., Leahy, K., Bruton, K., \& O’Sullivan, D. T. J. (2015). An industrial big data pipeline for datadriven analytics maintenance applications in largescale smart manufacturing facilities. Journal of Big Data, 2(1), 25. doi:10.1186/s40537-015-0034-z

O’Donovan, P., Leahy, K., Bruton, K., \& O’Sullivan, D. T. J. (2015). Big data in manufacturing: a systematic mapping study. Journal of Big Data, 2(1), 20. doi:10.1186/s40537-015-0028-x

Philip Chen, C. L., \& Zhang, C.-Y. (2014). Data-intensive applications, challenges, techniques and technologies: A survey on Big Data. Information Sciences, 275, 314-347. doi:10.1016/j.ins.2014.01.015

Reinisch, C., Granzer, W., Praus, F., \& Kastner, W. (2008). Integration of heterogeneous building automation systems using ontologies. 2008 34th Annual Conference of IEEE Industrial Electronics, 27362741. doi:10.1109/IECON.2008.4758391

Samad, T., \& Frank, B. (2007). Leveraging the web: a universal framework for building automation. In Proceedings of the 2007 American Control Conference.

Scholten, B. (2007). Integrating ISA-88 and ISA-95. In ISA Expo (pp. 2-4).
Sharma, P., \& Sharma, M. (2014). Artificial Intelligence in Advance Manufacturing Technology-A Review Paper on Current Application. International Journal of Engineering, Management \& Sciences, 1(1), 4-7.

Vera-baquero, A., Colomo-palacios, R., \& Molloy, O. (2014). Towards a process to guide Big Data based Decision Support Systems for Business Processes. In Conference on ENTERprise Information Systems Towards (Vol. 00).

Vincent Wang, X., \& Xu, X. W. (2013). An interoperable solution for Cloud manufacturing. Robotics and Computer-Integrated Manufacturing, 29(4), 232-247. doi:10.1016/j.rcim.2013.01.005

Weiss, B. A., \& Donmez, M. A. (2014). Standards Related to Prognostics and Health Management (PHM ) for Manufacturing Standards Related to Prognostics and Health Management (PHM ) for Manufacturing. In Annual Conference of the Prognostics and Health Management Society. doi:10.6028/NIST.IR.8012

Wright, P. (2014). Cyber-physical product manufacturing. Manufacturing Letters, 2(2), 49-53. doi:10.1016/j.mfglet.2013.10.001

Wu, D., Greer, M. J., Rosen, D. W., \& Schaefer, D. (2013). Cloud manufacturing: Strategic vision and state-ofthe-art. Journal of Manufacturing Systems, 32(4), 564-579. doi:10.1016/j.jmsy.2013.04.008

$\mathrm{Xu}, \quad \mathrm{X}$. (2012). From cloud computing to cloud manufacturing. Robotics and Computer-Integrated Manufacturing, 28(1), 75-86. doi:10.1016/j.rcim.2011.07.002

Zuehlke, D. (2010). SmartFactory-Towards a factory-ofthings. Annual Reviews in Control, 34(1), 129-138. doi:10.1016/j.arcontrol.2010.02.008 
APPENDIX A

\begin{tabular}{l|l}
\hline Abbreviation & Description \\
\hline AHU & Air Handling Unit \\
\hline AWS & Amazon Web Services \\
\hline BMS & Building Management System \\
\hline CPS & Cyber Physical Systems \\
\hline EC2 & Elastic Cloud Compute \\
\hline HTTP & Hypertext Transfer Protocol \\
\hline IT & Information Technology \\
\hline OPC & OLE Process Control \\
\hline OT & Operation Technology \\
\hline PC & Personal Computer \\
\hline PLC & Programmable Logic Controller \\
\hline PMML & Predictive Modeling Markup Language \\
\hline REST & Representational State Transfer \\
\hline S3 & Simple Storage Service \\
\hline SOA & Service Oriented Architecture \\
\hline SOAP & Simple Object Access Protocol \\
\hline SQS & Simple Queue Service \\
\hline SVM & Support Vector Machine
\end{tabular}

